TITLE:

\title{
Short-Time-Scale Processes in a Mature Hurricane as a Response to Sea Surface Fluctuations
}

\section{$\operatorname{AUTHOR}(\mathrm{S}):$}

Ito, Kosuke; Ishikawa, Yoichi; Miyamoto, Yoshiaki; Awaji, Toshiyuki

\section{CITATION:}

Ito, Kosuke ... [et al]. Short-Time-Scale Processes in a Mature Hurricane as a Response to Sea Surface Fluctuations. Journal of the Atmospheric Sciences 2011, 68(10): 2250-2272

\section{ISSUE DATE:}

2011-10

URL:

http://hdl.handle.net/2433/158038

RIGHT:

(C) 2011 merican Meteorological Society 


\title{
Short-Time-Scale Processes in a Mature Hurricane as a Response to Sea Surface Fluctuations
}

\author{
Kosuke ITO, Yoichi IsHikawa, AND Yoshiaki MiYAmoto \\ Kyoto University, Kyoto, Japan \\ TOSHIYUKI AWAJI \\ Data Research Center for Marine-Earth Sciences, JAMSTEC, Yokohama, and Kyoto University, Kyoto, Japan
}

(Manuscript received 8 December 2010, in final form 21 April 2011)

\begin{abstract}
To clarify the effect of fluctuations in surface stress and heat fluxes on the intensity of a mature-state hurricane, a sensitivity analysis is performed by using a cloud-permitting nonhydrostatic axisymmetric adjoint model. The response function of our experiment is tangential velocity at the top of the boundary layer in the eyewall.

As a result of an integration backward to 4 min prior to the specified time, a dipole pattern appears in the sensitivity fields with respect to the vertical velocity, the potential temperature, and the mixing ratio of water vapor. A positive (negative) sensitivity is found in the hurricane interior (exterior) relative to the verification region. It exhibits an increase of tangential velocity $4 \mathrm{~min}$ after the introduction of positive (negative) perturbations in potential temperature or in the mixing ratio of water vapor in the interior (exterior). These sensitivities are not related to the changes in the central pressure field. With further backward integration, the sensitivity signals reach down to the surface and are located in the exterior region of the hurricane. While the sensitivity with respect to surface friction (heat flux) is strongly negative (positive) within a certain radius, the sensitivity can be positive (negative) beyond that radius. This means that both stronger friction and a reduction in moist air supply in the exterior region of the hurricane can serve to strengthen the maximum tangential velocity. To the authors' knowledge, this effect has not been explained in previous studies.
\end{abstract}

\section{Introduction}

Since hurricanes are often highly destructive, a better understanding of their intensity is important for scientific progress and disaster prevention. There are growing concerns about enhanced hurricane intensity due to global warming (Emanuel 2005; Yokoi and Takayabu 2009; Knutson et al. 2010a,b; Yamada et al. 2010) because the actual financial cost arising from the passage of a hurricane on land is thought to relate to the maximum wind speed through a power law (Southern 1979; Nordhaus 2010). However, changes in intensity are still challenging researchers because they are governed by a complex array of physical processes in the inner core and depend on environmental factors such as underlying

Corresponding author address: Kosuke Ito, Department of Geophysics, Graduate School of Science, Kyoto University, KitashirakawaOiwake-Cho, Sakyo-Ku, Kyoto 606-8502, Japan.

E-mail: itokosk@kugi.kyoto-u.ac.jp oceanic features and a vertical wind shear (e.g., Schade and Emanuel 1999; Frank and Ritchie 2001; Wang and Wu 2004; Bender et al. 2007; Lloyd and Vecchi 2011).

It has been proposed that a hurricane vortex can intensify and maintain the primary circulation against surface friction through self-inducement of anomalous fluxes of moist enthalpy from the sea surface (Emanuel 1986). This model has been widely accepted for over $20 \mathrm{yr}$ with some modifications (Emanuel 1995; Bister and Emanuel 1998). As in these works, the maximum tangential velocity at the top of the boundary layer in the eyewall of an axisymmetric vortex has been used because it represents one of the key measures of hurricane intensity.

Although a (quasi-) steady-state axisymmetric vortex case has been intensively investigated to explain the maximum tangential velocity in theoretical (Emanuel 1986, 1995; Bister and Emanuel 1998; Smith et al. 2008; Bryan and Rotunno 2009c) and numerical modeling studies (Rotunno and Emanuel 1987; Persing and Montgomery 
2003; Hausman et al. 2006; Bryan and Rotunno 2009a,b,c), in reality the storm often encounters small-scale oceanic variability due to swells, oceanic mesoscale eddies, and western boundary currents. Counter (following) swells act to further increase (decrease) surface drag (Donelan et al. 1997; Drennan et al. 1999; Suzuki et al. 2010). Furthermore, warm (cold) mesoscale eddies act to enhance (degrade) latent and sensible heat fluxes at the sea surface (Lin et al. 2005; Wu et al. 2007b). According to the energy balance obtained from the current state-ofthe-art theoretical framework, these oceanic factors can potentially have an impact on tangential maximum velocity via changes in air-sea momentum and sensible and latent heat fluxes (Emanuel et al. 2004; Lin et al. 2005). Thus, an accurate formulation for the time evolution of these oceanic fluctuations is required for further understanding of hurricane intensity.

One of the fundamental questions is how perturbations of momentum and sensible and latent heat fluxes bring about changes in the maximum tangential velocity of hurricanes. In terms of the perturbation of the latent heat fluxes at sea surface, one may identify changes in the central pressure field as a mechanism capable of intensifying the hurricane vortex after the enhancement of condensation in the eyewall. However, Wu et al. (2006) showed that perturbation-like inputs to the central pressure field are not likely to affect the subsequent maximum tangential velocity substantially when the radius of the eyewall is smaller than the Rossby deformation radius, which is typically the case in the mature stage. This implies that the scenario in which an increase in condensation contributes to changes in the maximum tangential velocity following a decrease in the central pressure field is an inadequate description of the time-dependent behavior.

In this study, we perform a sensitivity analysis by using a cloud-permitting nonhydrostatic axisymmetric adjoint model to examine the role of sea surface fluctuations in determining the intensity of a mature-state hurricane. The adjoint-based analysis can trace the sensitivity of the response function backward in time, as is well known in data assimilation. Here, we take the tangential velocity at the top of the boundary layer as a response function and trace back the sensitivity. The adjoint-based sensitivity analysis has several advantages over other methods. First, all the sensitivities associated with the response function are obtained by one-time backward integration (here, the original nonlinear model is termed the forward model). Compared with another type of experiment in which all the model variables are perturbed, this method is efficient and offers an integrated point of view. Second, the propagation of sensitivity signals exhibits all processes corresponding to the terms in the forward model.
Therefore, the adjoint-based sensitivity fields reflect the physical processes associated with wave propagation, advection, and so on. Furthermore, we can divide changes in sensitivity fields into the contributions of each physical process by the term balance analysis of the adjoint equation.

Although the adjoint-based sensitivity analysis has previously been applied to the tropical cyclogenesis (Doyle et al. 2010) and the steering flow of a hurricane (Wu et al. 2007a), this method has not been fully applied to numerical study of a mature hurricane with fine grid spacing. We therefore perform a finescale adjoint-based sensitivity analysis to further our understanding of hurricane inner core dynamics and thermodynamics and their relationship to the variability at the sea surface. Of course, a limitation arises from the nonlinear nature of the system and our simplification of the model dynamics. Nevertheless, this study contributes to resolving the complex array of physics and, in particular, to accounting for the time-dependent change in hurricane intensity.

The rest of this paper is organized as follows. Section 2 outlines the adjoint-based sensitivity analysis. An experimental design is given in section 3 with a description of the forward model, tangent linear model, and adjoint model. In sections 4 and 5, the sensitivity of the maximum tangential velocity within the verification region with respect to the state variables and air-sea fluxes is considered. We present some discussion in section 6 , while our conclusions are summarized in section 7 .

\section{Theoretical background}

The adjoint-based sensitivity analysis is outlined in this section. A more detailed explanation is given in Lewis et al. (2006, 382-421). Consider a system in which the time evolution of the prognostic state variables can be expressed as

$$
\mathbf{x}_{m+1}=\mathcal{M}_{m}\left(\mathbf{x}_{m} ; \mathbf{c}_{m}\right),
$$

where we denote a prognostic state variable at the discretized individual time step $m$ as $\mathbf{x}=\mathbf{x}_{m}$, a model parameter as $\mathbf{c}=\mathbf{c}_{m}$, and an operation that advances the state variable by one discretized time step as $\mathcal{M}_{m}$. When the perturbations of the state variables applied at discretized time step $m$ are represented as $\delta \mathbf{x}=\delta \mathbf{x}_{m}$ and those of the model parameter as $\delta \mathbf{c}=\delta \mathbf{c}_{m}$, a first-order Taylor series approximation with respect to the independent variables $\mathbf{x}_{m}$ and $\mathbf{c}_{m}$ provides the time evolution of perturbation as follows:

$$
\delta \mathbf{x}_{m+1}=\mathbf{M}_{m} \delta \mathbf{x}_{m}+\mathbf{F}_{m} \delta \mathbf{c}_{m},
$$


where $\mathbf{M}_{m}\left(\equiv \partial \mathcal{M}_{m} / \partial \mathbf{x}\right)$ and $\mathbf{F}_{m}\left(\equiv \partial \mathcal{M}_{m} / \partial \mathbf{c}\right)$ are the firstorder partial derivatives of vectors about $\mathbf{x}=\mathbf{x}_{m}$ and $\mathbf{c}_{m}=$ $\mathbf{c}_{m}$. The operator $\mathbf{M}_{m}$ is hereafter referred to as the tangent linear operator. In this study, $\mathbf{F}_{m}$ represents the influence of parameters at the sea surface on model variables in the lowest atmospheric layer.

Then, we define the response function $J=J\left(x_{n}\right)$, which is a scalar function calculated by the variables at the discretized individual time step $n(>m)$ of the forward calculation. By considering the total differential of this function with respect to the independent variables $\mathbf{x}_{m}$ and $\mathbf{c}_{m}$,

$$
\delta J\left(x_{n}\right)=\boldsymbol{\lambda}_{m}^{\mathbf{x}} \cdot \delta \mathbf{x}_{m}+\boldsymbol{\lambda}_{m}^{\mathbf{c}} \cdot \delta \mathbf{c}_{m},
$$

where $\boldsymbol{\lambda}_{m}^{\mathbf{x}} \equiv \partial J / \partial \mathbf{x}_{m}$ and $\boldsymbol{\lambda}_{m}^{\mathbf{c}} \equiv \partial J / \partial \mathbf{c}_{m}$; the dot represents the inner product and $\delta$ indicates the perturbation from the reference fields. Hereafter $\boldsymbol{\lambda}_{m}^{\mathbf{x}}$ and $\boldsymbol{\lambda}_{m}^{\mathbf{c}}$ are referred to as sensitivities with respect to the state variables and parameter values or simply "sensitivity," since $\boldsymbol{\lambda}_{m}^{\mathbf{x}}$ and $\boldsymbol{\lambda}_{m}^{\mathbf{c}}$ can be interpreted as the changes in $J$ in response to infinitesimal perturbations of $\mathbf{x}_{m}$ and $\mathbf{c}_{m}$.

Some calculus yields the following equation for the sensitivity with respect to the state variables:

$$
\boldsymbol{\lambda}_{m}^{\mathbf{x}}=\mathbf{M}_{m}^{*} \boldsymbol{\lambda}_{m+1}^{\mathbf{x}}
$$

The matrix $\mathbf{M}_{m}^{*}$ represents the adjoint of $\mathbf{M}_{m}$. This equation expresses backward integration in the sense that a gradient with respect to the state variables at $m$ is obtained from operating the adjoint matrix on the gradient with respect to these variables at the discretized individual time step $m+1$ (Errico 1997). Note that Eq. (4) is valid only if the time evolution of the perturbations in a nonlinear forward model is well approximated by that in a tangent linear model.

In this study, the sensitivity for parameters such as surface fluxes and sea surface temperature (SST) from discretized individual time step $m$ to $m^{\prime}\left(n>m^{\prime}>m\right)$ is calculated as an accumulated value of $\mathbf{F}_{m}^{*} \boldsymbol{\lambda}_{m+1}^{\mathbf{x}}$ as follows:

$$
\boldsymbol{\lambda}^{c}=\sum_{i=m}^{m^{\prime}} \mathbf{F}_{i}^{*} \boldsymbol{\lambda}_{i+1}^{\mathbf{x}},
$$

where $i$ represents the discretized individual time step and $\mathbf{F}_{i}^{*}$ is the adjoint operator of $\mathbf{F}_{i}$. As indicated by this equation, sensitivity with respect to parameter values is calculated by using output from the backward integration $\boldsymbol{\lambda}_{i+1}^{\mathbf{x}}$. More specifically, $\mathbf{F}_{i}^{*}$ used here works to transforms the sensitivity with respect to the model variables in the lowest atmospheric layer into the sensitivity with respect to the parameter values at the surface boundary.

In this study, all the adjoint operators $\mathbf{M}^{*}$ are defined with respect to the Euclidean inner product and all variables are real numbers. In this case, an adjoint operator is expressed simply by the transpose of the corresponding tangent linear matrix $\mathbf{M}^{\mathrm{T}}$.

\section{Experimental design}

\section{a. Forward model}

We employ the nonhydrostatic, axisymmetric, cloudpermitting atmospheric model on an $f$ plane as formulated by Rotunno and Emanuel (1987). The version with improved energy conservation (K. Emanuel 2008, personal communication) is used here along with a thirdorder upwind advection scheme, but this version does not include the process of dissipative heating proposed by Bister and Emanuel (1998). The governing equations are summarized in the appendix [Eqs. (A1)-(A7)]. The operator $\mathcal{M}_{m}$ used in this study consists of the discretized version of the governing equations. In brief, the nonhydrostatic, compressible equation of motion is integrated in time with the prognostic equations for radial velocity $u$, tangential velocity $v$, vertical velocity $w$, potential temperature $\theta$, nondimensional pressure $\pi$, and mixing ratios of water vapor $q$ and of liquid water $q_{\text {liq }}$. To accommodate rapidly propagating sound waves, a few terms associated with the pressure tendency are computed with a "small" time step, while the rest are computed with a long time step (Klemp and Wilhelmson 1978).

This intermediate model is thought to capture the axisymmetric characteristics of a hurricane such as prominent tangential velocity, inward (outward) flow in the boundary layer (upper troposphere), vertical velocity associated with the eyewall, and warm core in the upper portion of hurricane center. Needless to say, the primary drawback of axisymmetric models is that they inevitably lack threedimensional features such as mesovortices around the inner core region, boundary layer roll vortices, upper-level asymmetric outflow jets, vortex Rossby waves, and so on. Our intent is not to replicate a realistic evolution but rather to elucidate the fundamental dynamics responsible for the impact of sea surface fluctuations.

\section{b. Experimental setting for a forward model}

We employ the same sounding shown in Fig. 1a of Bryan and Rotunno (2009a). The long time step of $0.2 \mathrm{~s}$ (and small time step of $0.04 \mathrm{~s}$ ) used here are set smaller than in previous studies. With this configuration, the discrepancy between the nonlinear forward model and 
the tangent linear model diminishes (Xu 1997). Since the error in the adjoint-based sensitivity arises from the unreasonable linearization of the time evolution of the perturbation, this makes the adjoint-based sensitivity more appropriate. Otherwise, the experimental settings are the same as those used in the " $4 \times$ run" of Persing and Montgomery (2003). The domain has $400 \times 80$ grids with a radial grid spacing of $3.75 \mathrm{~km}$ and vertical grid spacing of $312.5 \mathrm{~m}$. The vortex is given on day 0.0 with a maximum tangential velocity of $12.7 \mathrm{~m} \mathrm{~s}^{-1}$ at a radius $r$ taken as $82.5 \mathrm{~km}$ from the center. The SST field of the forward run is fixed to be $299.28 \mathrm{~K}\left(=26.13^{\circ} \mathrm{C}\right)$ as used in the control run of Bryan and Rotunno (2009a).

The magnitude of surface fluxes are given as

$$
\begin{aligned}
& \boldsymbol{\tau}_{r z}(r)=-\rho C_{D}\left|\mathbf{V}_{l}\right| u_{l}, \\
& \boldsymbol{\tau}_{\phi z}(r)=-\rho C_{D}\left|\mathbf{V}_{l}\right| v_{l}, \\
& \mathbf{F}_{H}(r)=-\rho C_{p} C_{H}\left|\mathbf{V}_{l}\right|\left(\theta_{l}-\theta_{s}\right),
\end{aligned}
$$

and

$$
\mathbf{F}_{E}(r)=-\rho L C_{E}\left|\mathbf{V}_{l}\right|\left(q_{l}-q_{\mathrm{vsw}}\right) .
$$

Here, $\boldsymbol{\tau}_{r z}$ and $\boldsymbol{\tau}_{\phi z}$ are the radial and tangential surface stress, $\mathbf{F}_{H}$ and $\mathbf{F}_{E}$ are sensible and latent heat fluxes across the sea surface, respectively, $\rho$ is the density, $C_{p}$ is the heat capacity of air, $L$ is the latent heat of condensation per unit mass, $q_{\mathrm{vsw}}$ is the saturated mixing ratio of water vapor at the sea surface, and $|\mathbf{V}|=\sqrt{u^{2}+v^{2}}$. The subscripts $l$ and $s$ represent the corresponding values at the lowest layer of the atmosphere and the sea surface, respectively. The coefficients $C_{D}, C_{H}$, and $C_{E}$ are bulk coefficients for momentum, sensible heat, and latent heat exchange, respectively.

\section{c. Forward model and tangent linear model run}

The maximum tangential velocity obtained in this run is shown in Fig. 1. The vortex develops after 4 days and in terms of intensity, it appears to maintain a quasisteady state after 7 days. We now compare the time evolutions of the initial perturbations obtained from the nonlinear forward model and from the tangent linear model run in order to determine the period over which the linearization provides an acceptable description. Here we introduce a small perturbation of $v$ on day $10.0(240.0 \mathrm{~h})$ with a magnitude of $10^{-8} \mathrm{~m} \mathrm{~s}^{-1}$ at six grid points upstream of the hurricane inner core as in Figs. 2a and 2e: $(r, z)=(50.625 \mathrm{~km}, 156.25 \mathrm{~m}),(50.625 \mathrm{~km}$, $468.75 \mathrm{~m}),(54.375 \mathrm{~km}, 156.25 \mathrm{~m}),(54.375 \mathrm{~km}, 468.75 \mathrm{~m})$, $(58.125 \mathrm{~km}, 156.25 \mathrm{~m})$, and $(58.125 \mathrm{~km}, 468.75 \mathrm{~m})$. While

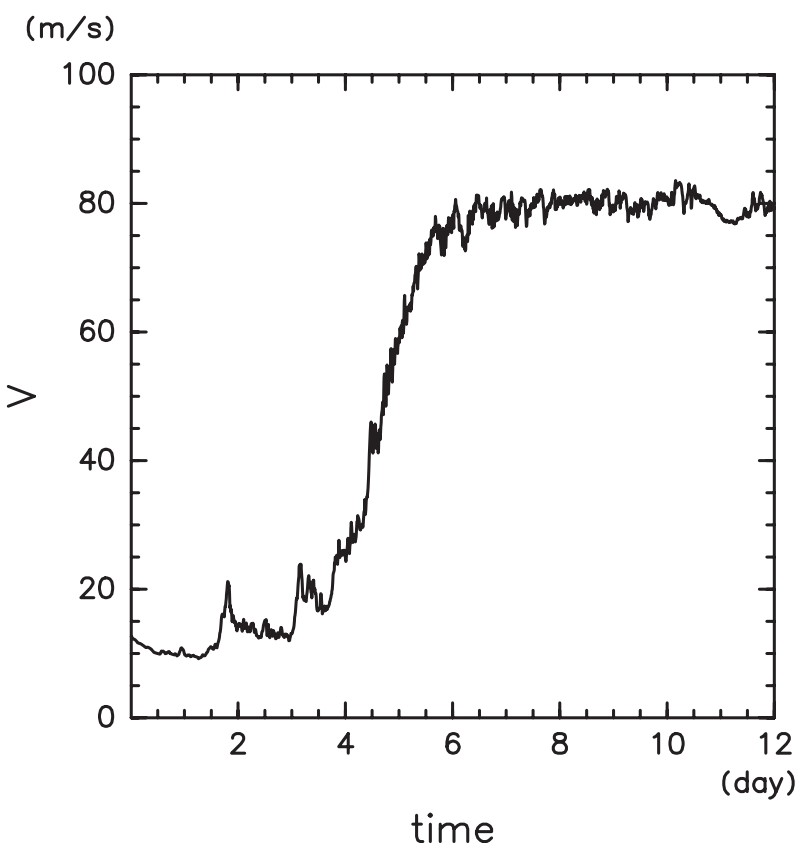

FIG. 1. Time series of the maximum tangential velocity.

in general the perturbation could be introduced to any grid points, the points were chosen to be upstream of the hurricane inner core because the time evolution of the perturbation in the inner core is a vital part in this study.

Figure 2 shows that the time evolution of the perturbation obtained from the forward model is well approximated by tangent linear model for a period of 60 min (see Fig. 3 for the reference wind field). The differences in the time evolution between the forward model and the tangent linear model are discernible after this period. The discrepancy is presumably associated with the linearization of, for example, discontinuities in the moist processes [see Xu $(1996,1997)$ for mathematical details]. The valid time scale of tangent linear assumption is extended to about $2 \mathrm{~h}$ with moist processes switched off (not shown). However, we include the moist processes in the adjoint equation to retain all the physical possibilities. Thus, we do not discuss the calculation with moist processes switched off hereafter.

As expected, the time scale relevant to the linear development of a perturbation in a hurricane inner core is rather short given the nature of the system. We have carried out the experiment to clarify whether an hour is fit for our purpose of investigating the cause-and-effect series of events associated with fluctuations in the airsea fluxes. By doubling the magnitude of the air-sea momentum and latent heat exchange coefficients within a radius of $100 \mathrm{~km}$, tangential wind speed varies by 
(a) $\mathrm{NL}(\mathrm{t}=20 \mathrm{~min})$

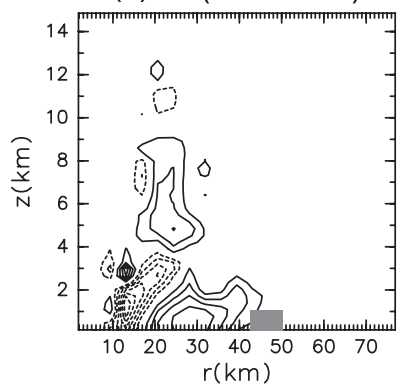

(e) $\mathrm{TL}(\mathrm{t}=20 \mathrm{~min})$

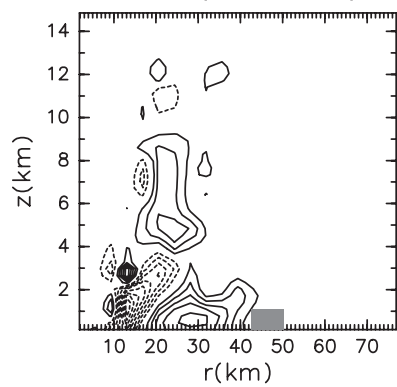

(b) $\mathrm{NL}(\mathrm{t}=40 \mathrm{~min})$

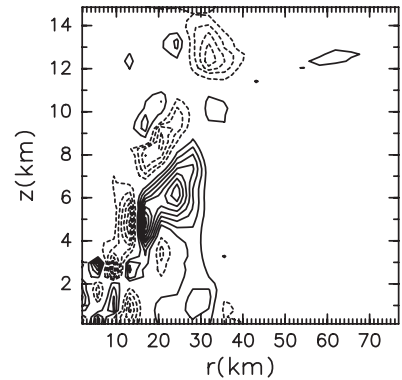

(f) $T L(t=40 \mathrm{~min})$

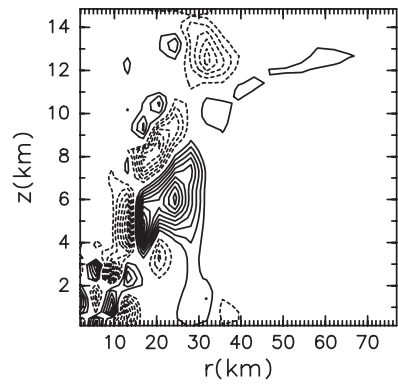

(c) $\mathrm{NL}(\mathrm{t}=60 \mathrm{~min})$

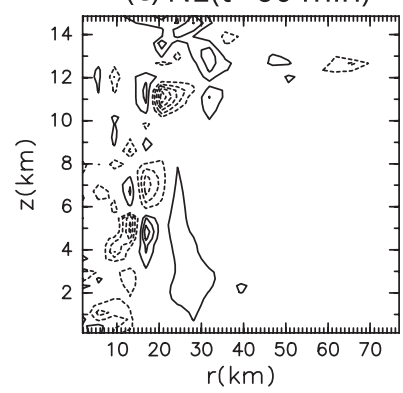

(g) $\mathrm{TL}(\mathrm{t}=60 \mathrm{~min})$

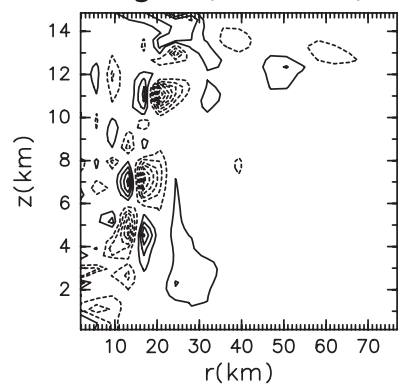

(d) $\mathrm{NL}(\mathrm{t}=80 \mathrm{~min})$

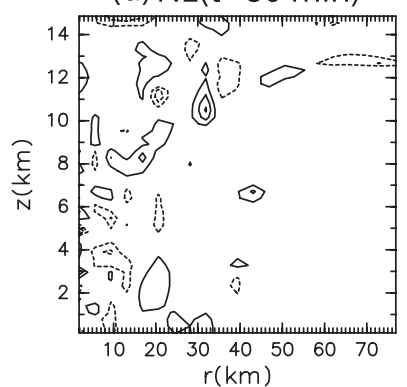

(h) TL(t=80 $\mathrm{min})$

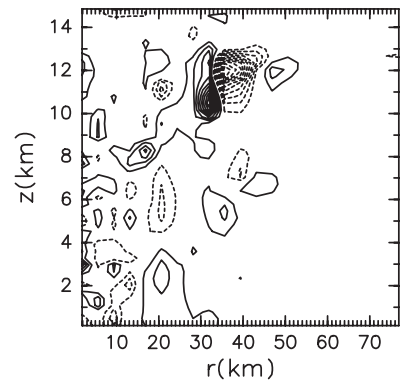

FIG. 2. Time evolution of the initial perturbations derived by the nonlinear forward model (NL) and the tangent linear model (TL) from 20 to $80 \mathrm{~min}$. The number of minutes beginning at $240.0 \mathrm{~h}$ is used here. Contours are $\delta v$ every $8.0 \times 10^{-10} \mathrm{~m} \mathrm{~s}^{-1}$ with zero contour excluded.

-6.20 and $3.12 \mathrm{~m} \mathrm{~s}^{-1}$ within an hour, respectively (not shown). Taking into account these facts, a period of $60 \mathrm{~min}$ for backward integration (from 241.0 to $240.0 \mathrm{~h}$ ) seems appropriate for this sensitivity analysis. Note that study of the time evolution for longer time periods is beyond the scope of the present approach. For the presentation of results, the number of minutes beginning at $241.0 \mathrm{~h}$ (a minus sign indicates a backward temporal shift) is used unless noted otherwise. We refer to $241.0 \mathrm{~h}$ to as the specified time. The time interval of the output is $15 \mathrm{~s}$. To obtain the sensitivity fields by backward integration, the output of forward calculations during this period are memorized at every step and they are used as the reference field.

The time-mean wind fields from the initial time of the backward integration to $-60 \mathrm{~min}$ (at the final time of backward integration) are shown in Fig. 3a. The wind fields clearly capture the fundamental characteristics of the hurricane. The component of $v$ is characterized by a sharp maximum, whereas the $u$ field shows an inward (outward) flow in the boundary layer (upper troposphere). Inflow is also seen around $z=8 \mathrm{~km}$ outside the eyewall $(r>30 \mathrm{~km})$. The vertical velocity indicates the formation of the eyewall encircling the eye. Figures $3 b-d$ show snapshots of wind fields at $-10,-30$, and $-50 \mathrm{~min}$. The location of maximum tangential velocity is maintained at $r=15 \mathrm{~km}$ and $z=1.5 \mathrm{~km}$ and the tangential velocity field seems to be in a quasi-steady state, while short-lived convective cells with downdrafts and updrafts are present outside the eyewall $(r>30 \mathrm{~km})$ from $z=2$ to $z=10 \mathrm{~km}$. The time-mean diabatic heating rate and the potential temperature deviation during the same period are shown in Fig. 4a. This figure also exhibits the fundamental characteristics of hurricane such as large diabatic heating rate in the eyewall and the formation of the warm core. Figures $4 \mathrm{~b}-\mathrm{d}$ support the presence of short-lived convective cells outside the eyewall $(r>30 \mathrm{~km})$.

\section{d. Adjoint model}

The adjoint model used in this study is an updated version of that used by Ito et al. (2010). The adjoint equations are summarized in the appendix [Eqs. (A8)(A14)]. They consist of the adjoint code of the forward model equation, although physical processes with parameterized discontinuities are treated as conventional on/off switches [see Xu (1997) for details] and eddy diffusivity calculated in the forward model is used for the adjoint calculation to avoid strong nonlinearity.

A response function used for the sensitivity analysis is defined as the integrated tangential velocity $\left(\mathrm{m} \mathrm{s}^{-1}\right)$ within the verification region $\mathcal{A}$ ranging from $r=15.0$ to $r=22.5 \mathrm{~km}$, and from $z=937.5$ to $z=2187.5 \mathrm{~m}$. More specifically, 
(a) $(u, v, w)$ : 1hour-mean

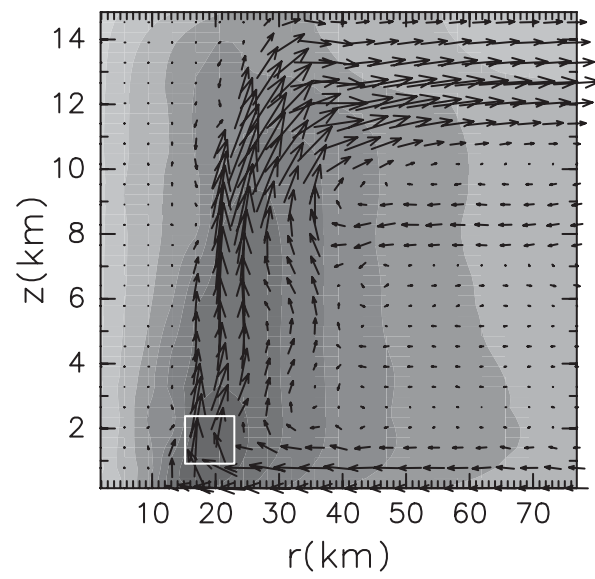

(c) $(u, v, w):-30$ min.

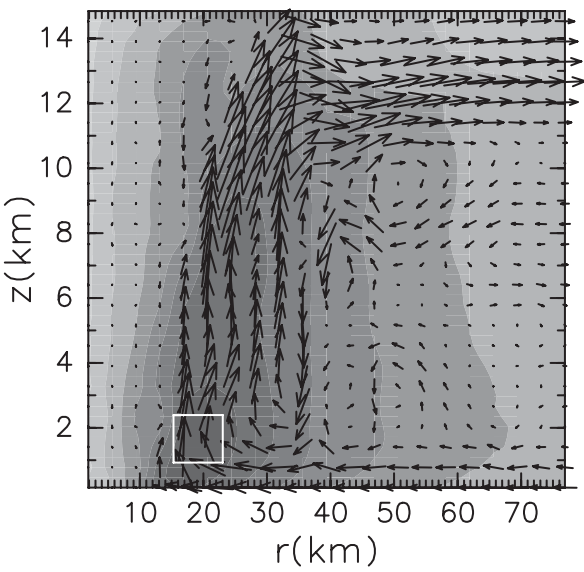

(b) $(u, v, w):-50 \mathrm{~min}$.

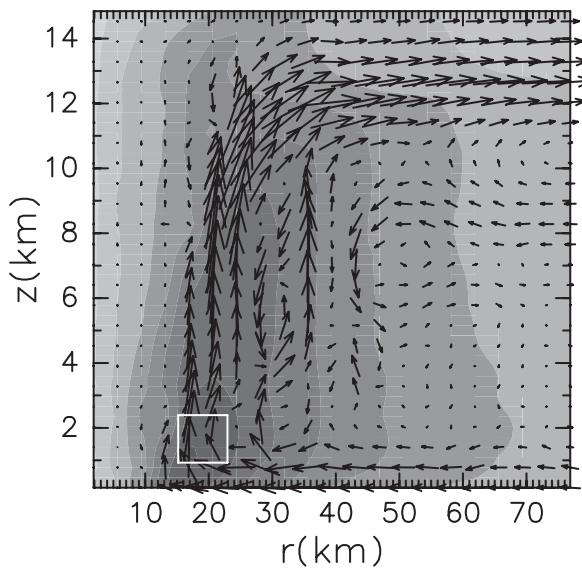

(d) $(u, v, w)$ : $-10 \mathrm{~min}$.

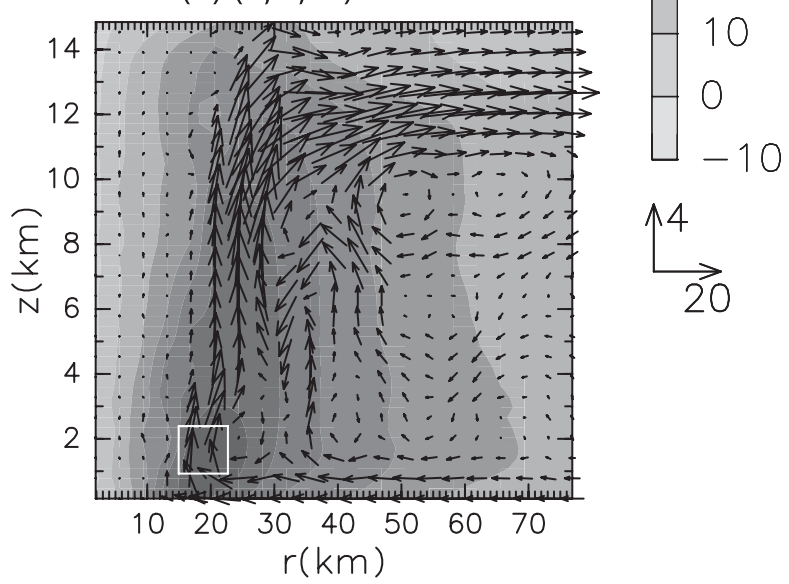

FIG. 3. Wind fields $\left(\mathrm{m} \mathrm{s}^{-1}\right)$ of the reference state, showing (a) the time mean between 0 and -60 min as well as snapshots at (b) -50 , (c) -30 , and (d) $-10 \mathrm{~min}$. Shading denotes tangential velocity. Radial velocity and vertical velocity (vectors) are superposed. The white rectangles indicate the verification region $\mathcal{A}$.

$$
J=\sum_{(r, z) \in \mathcal{A}} v(t=0) .
$$

Here, the number of grid points within the verification region $\mathcal{A}$ is 8 points: $(r, z)=(16.875 \mathrm{~km}, 1093.75 \mathrm{~m})$, $(16.875 \mathrm{~km}, 1406.25 \mathrm{~m}),(16.875 \mathrm{~km}, 1718.75 \mathrm{~m}),(16.875 \mathrm{~km}$, $2031.25 \mathrm{~m}),(20.625 \mathrm{~km}, 1093.75 \mathrm{~m}),(20.625 \mathrm{~km}, 1406.25 \mathrm{~m})$, $(20.625 \mathrm{~km}, 1718.75 \mathrm{~m})$, and $(20.625 \mathrm{~km}, 2031.25 \mathrm{~m})$. Thus, the verification region $\mathcal{A}$ is set as a rectangle to make interpretation easier and it contains the maximum grid points where the tangential velocity is beyond $70 \mathrm{~m} \mathrm{~s}^{-1}$ continually during this period.

In this study, the sensitivity is defined as the ratio of change in $J$ in response to infinitesimal perturbations, which are regarded as a "ring of change" at $r$. All prognostic state variables $\left(u, v, \mathrm{w}, \pi, \theta, q, q_{\text {liq }}\right)$ and some surface variables are taken as independent variables for the present sensitivity analysis. The surface variables are $\tau_{r z}, \boldsymbol{\tau}_{\phi z}$,
$\mathbf{F}_{H}, \mathbf{F}_{E}, \mathrm{SST}$, and air-sea exchange coefficients. The sensitivities with respect to these surface variables are calculated from the equations shown in the appendix [Eqs. (A15)-(A22)]. For the presentation of results, the sensitivity with respect to the surface variables is formulated as a response to the changes per minute (section 5) or to the changes during specified period (section 6b), and not just to the changes at one discretized time step $m$.

The initial values for backward integration are

$$
\lambda^{v}=\left(\frac{\partial J}{\partial v}\right)_{t=0}= \begin{cases}1.0, & (r, z) \in \mathcal{A} \\ 0.0, & (r, z) \notin \mathcal{A}\end{cases}
$$

and

$$
\boldsymbol{\lambda}^{u}=\boldsymbol{\lambda}^{w}=\boldsymbol{\lambda}^{\pi}=\boldsymbol{\lambda}^{\theta}=\boldsymbol{\lambda}^{q}=\boldsymbol{\lambda}^{q_{\text {liq }}}=0.0 .
$$


(a) d $\theta /$ dt \& $\Delta \theta$ : 1hour-mean

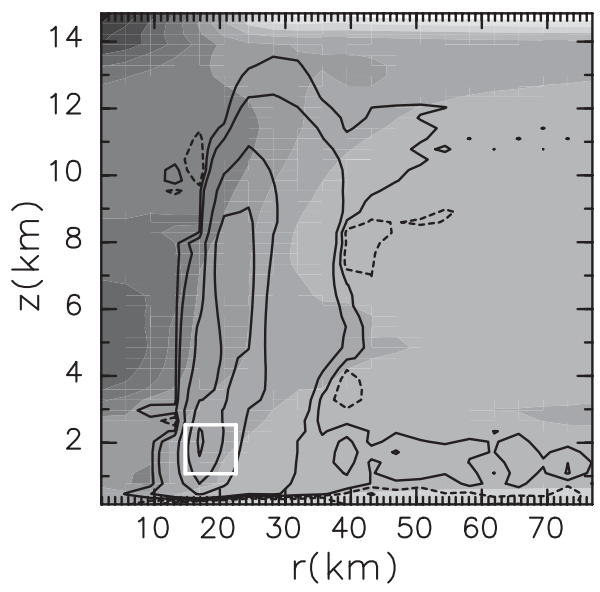

(c) $\mathrm{d} \theta / \mathrm{dt} \& \Delta \theta:-30 \mathrm{~min}$.

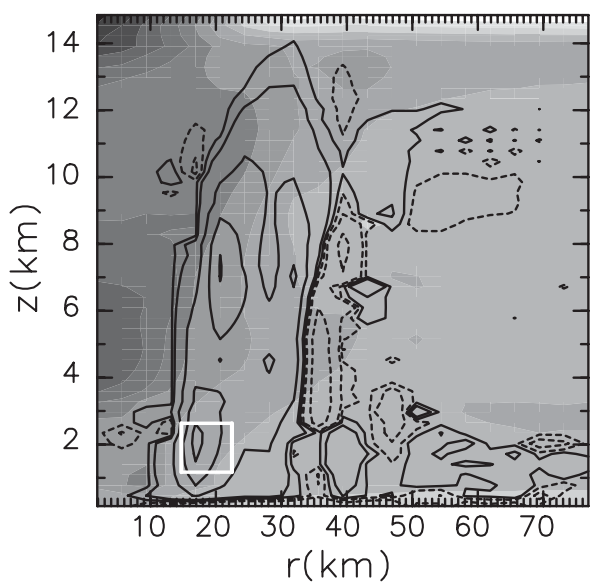

(b) d $\theta /$ dt \& $\Delta \theta:-50 \mathrm{~min}$.

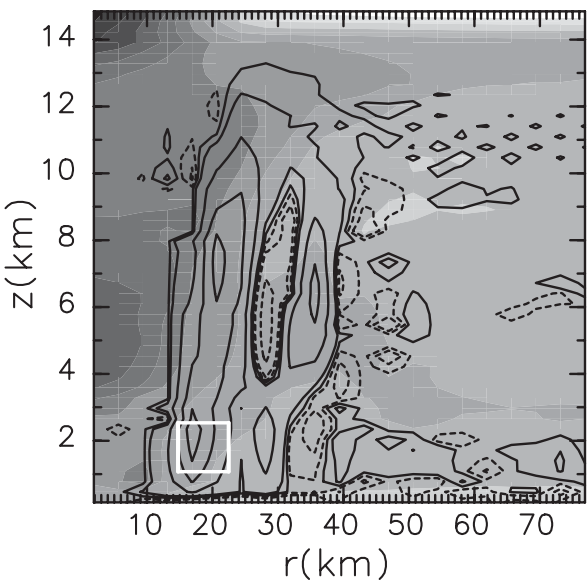

(d) $\mathrm{d} \theta / \mathrm{dt} \& \Delta \theta:-10 \mathrm{~min}$.

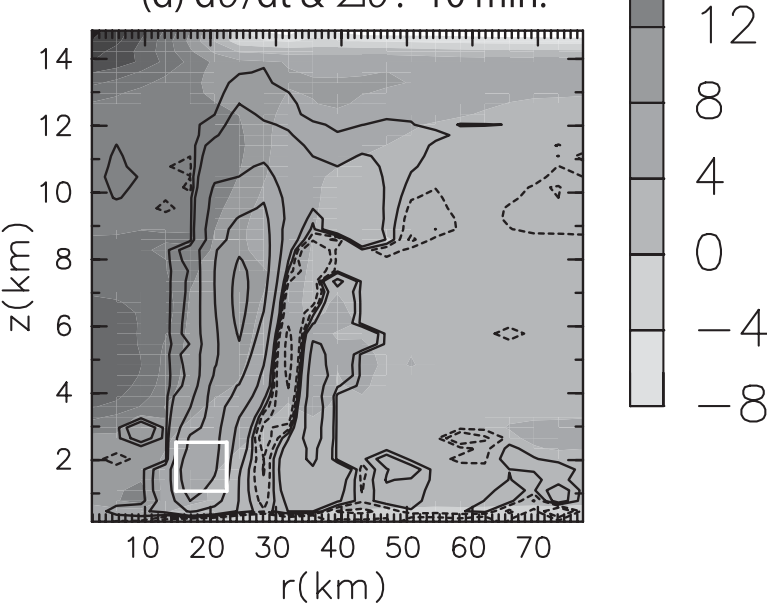

FIG. 4. Shading denotes potential temperature deviations $(\mathrm{K})$ from day 0 , while the contours denote the diabatic heating rate. The values of the shading are shown at right. Contour values are $\pm 5, \pm 20, \pm 80, \pm 160, \pm 240$, and $\pm 320 \mathrm{~K} \mathrm{~h}^{-1}$, with the zero contour excluded.

\section{Sensitivity with respect to state variables}

\section{a. Sensitivity at $-4 \mathrm{~min}$}

Figure 5 shows the sensitivity fields with respect to the state variables within $r<40 \mathrm{~km}$ and $z<6 \mathrm{~km}$ obtained by backward integration up to $-4 \mathrm{~min}$. According to the definition of sensitivity, $J$ is changed by $\boldsymbol{\lambda}^{x} \delta x$ at $t=0$ in the forward calculation when a small $\delta x$ is introduced at $-4 \mathrm{~min}$ in a given grid of the reference fields. It should be noted that the temporal change in $J$ depends not only on $\boldsymbol{\lambda}^{x}$ shown in Fig. 5 but also on the magnitude of $\delta x$.

Figure 5 exhibits several interesting features. 1) Negative values are seen in the adjoint variables $\boldsymbol{\lambda}^{u}$ around the verification region. The negative values of $\boldsymbol{\lambda}^{u}$ indicate that the tangential wind velocity increases at $0 \mathrm{~min}$ if we introduce a negative perturbation of $u$, namely stronger inward motion, in this region at $-4 \mathrm{~min}$. 2) The adjoint variables $\boldsymbol{\lambda}^{w}, \boldsymbol{\lambda}^{\theta}$, and $\boldsymbol{\lambda}^{q}$ show a deep dipole structure up to the height of $7 \mathrm{~km}$ and centered at $r=18 \mathrm{~km}$, whose positive (negative) values appear in the hurricane interior (exterior) relative to the verification region. Thus, the positive (negative) perturbations of these variables located in the interior (exterior) can locally intensify the tangential velocity in the verification region. 3) The sensitivity fields of $\boldsymbol{\lambda}^{q_{\text {liq }}}$ also have a dipole structure centered at the verification region but with reversed sign.

To explain these features, the term balances in the adjoint model [Eqs. (A8)-(A14)] along the solid lines in Fig. 5 around $z=1 \mathrm{~km}$ are calculated in order to examine the physical processes involved in the sensitivity fields (Fig. 6). Three-letter codes represent the terms in the adjoint model corresponding to those in the forward model (Table 1). Among these panels, we first focus on the changes in the sensitivity with respect to 
(a) Sensitivity: $\lambda_{u} @-4 \min$.

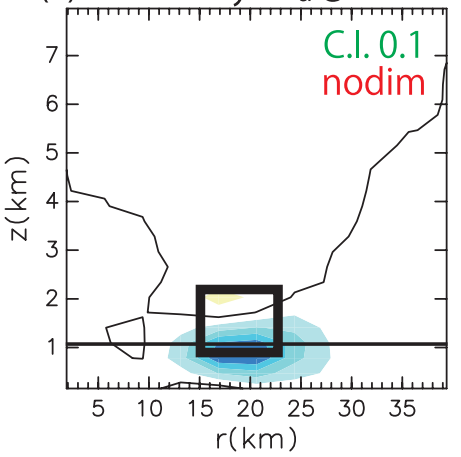

(c) Sensitivity: $\lambda_{w} @-4$ min

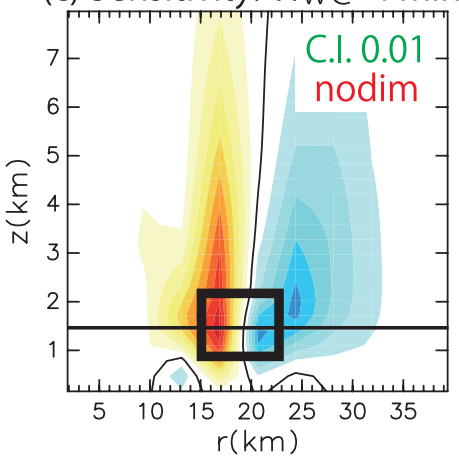

(e) Sensitivity: $\lambda_{\theta} @-4 \min$

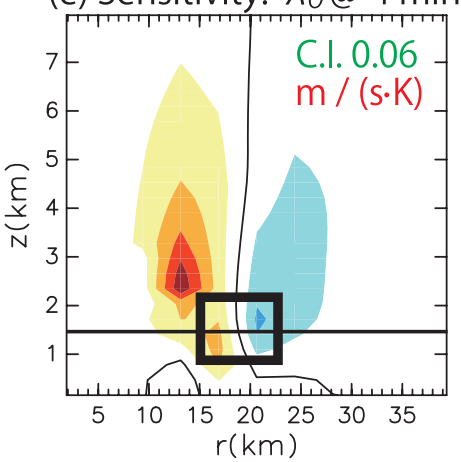

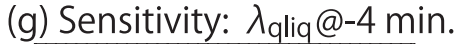

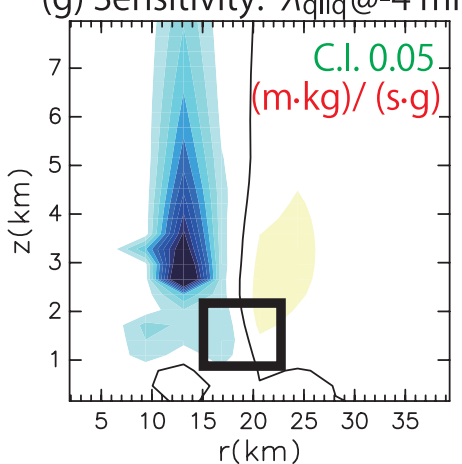

the wind fields (Figs. 6a-c). Note that the sensitivity fields of $\boldsymbol{\lambda}^{v}$ only affect the evolution of $\boldsymbol{\lambda}^{u}$ and $\boldsymbol{\lambda}^{w}$ via the adjoint equations. Figures $6 \mathrm{a}$ and $6 \mathrm{~b}$ indicate that the changes in $\boldsymbol{\lambda}^{u}$ and $\boldsymbol{\lambda}^{v}$ mainly correspond to the metric (b) Sensitivity: $\lambda_{\mathrm{v}} @-4 \min$.

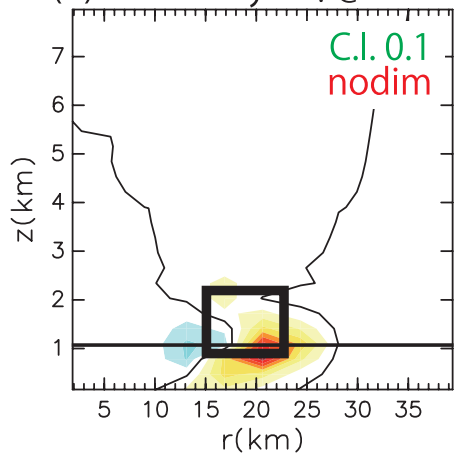

(d) Sensitivity: $\lambda_{\pi} @-4$ min.

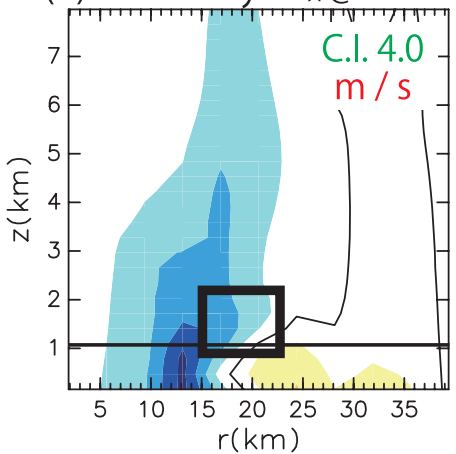

(f) Sensitivity: $\lambda_{q} @-4 \min$.

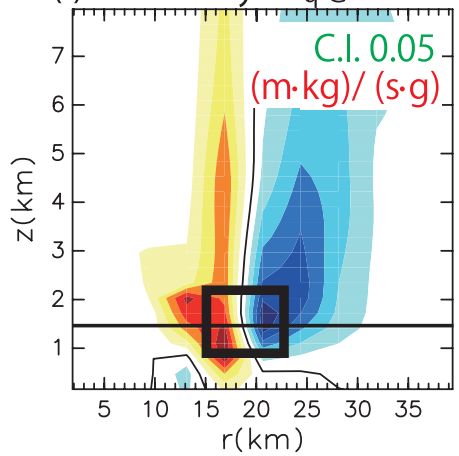

FIG. 5. The sensitivity of $J$ with respect to the field of (a) $u\left(\mathrm{~m} \mathrm{~s}^{-1}\right)$, (b) $v\left(\mathrm{~m} \mathrm{~s}^{-1}\right)$, (c) $w\left(\mathrm{~m} \mathrm{~s}^{-1}\right)$, (d) $\pi$, (e) $\theta(\mathrm{K}),(\mathrm{f}) q\left(\mathrm{~g} \mathrm{~kg}^{-1}\right)$, and (g) $q_{\text {liq }}$ $\left(\mathrm{g} \mathrm{kg}^{-1}\right)$ at -4 min. Solid lines indicate the altitudes where we consider the balances of terms in Fig. 6 . Contour intervals are indicated by numbers in green. The units of the sensitivity fields are shown in each panel. "Nodim" represents nondimensional variables. The rectangle in each panel indicates the verification region. terms $v^{2} / r$ and $-u v / r$ in the forward model [Eqs. (A1) and (A2)].

The advection term and terms associated with the small time step mainly contribute to the changes in $\boldsymbol{\lambda}^{w}$. 
(a) Term Balance: $\lambda_{\mathrm{u}}(-4 \mathrm{~min} .<\mathrm{t}<0$ min. $)$

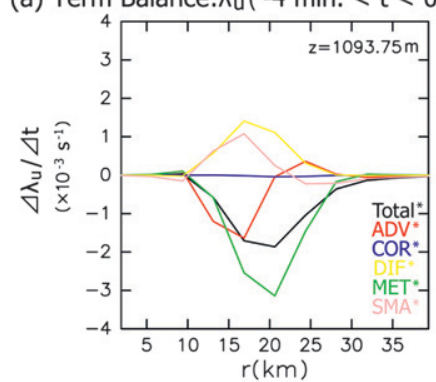

(d) Term Balance: $\lambda_{\pi}(-4$ min. $<\mathrm{t}<0$ min. $)$

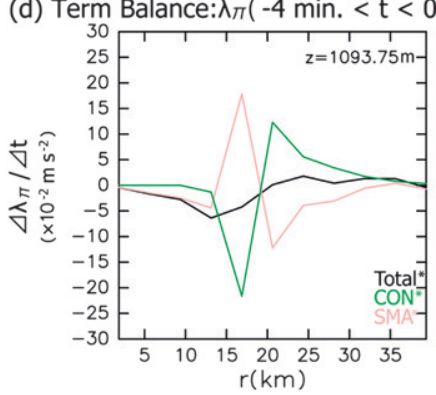

(b) Term Balance: $\lambda_{v}(-4$ min. $<\mathrm{t}<0$ min.)

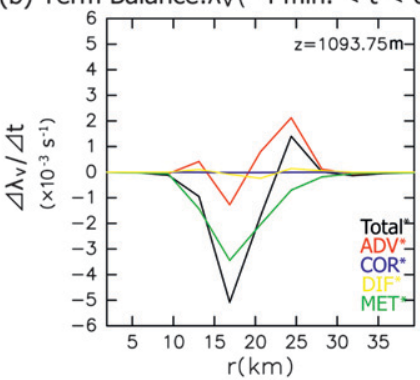

(e) Term Balance: $\lambda_{\theta}(-4 \min .<\mathrm{t}<0$ min.)

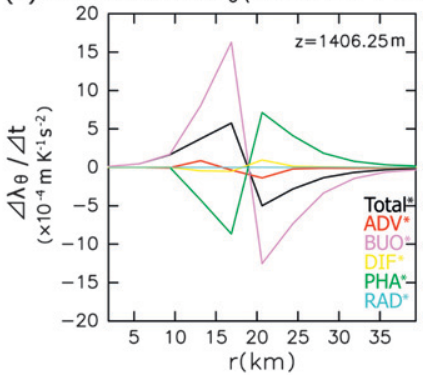

(c) Term Balance: $\lambda_{\mathrm{w}}(-4$ min. $<\mathrm{t}<0$ min. $)$

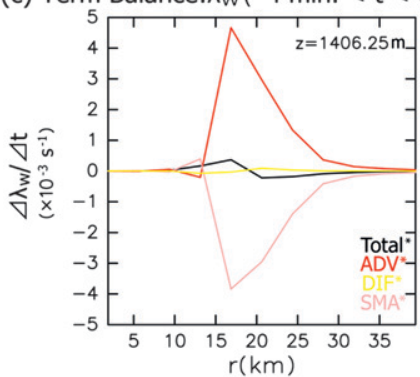

(f) Term Balance: $\lambda_{q}(-4$ min. $<\mathrm{t}<0$ min. $)$

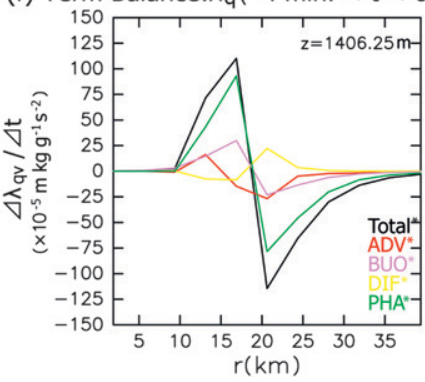

FIG. 6. Term balances in $\boldsymbol{\lambda}^{u}, \boldsymbol{\lambda}^{v}$, and $\boldsymbol{\lambda}^{\pi}$ at $z=1093.75 \mathrm{~m}$, and $\boldsymbol{\lambda}^{w}, \boldsymbol{\lambda}^{\theta}$, and $\boldsymbol{\lambda}^{q}$ at $z=1406.25 \mathrm{~m}$ between -4 and $0 \mathrm{~min}$.

Comparison of the sensitivity fields of $\boldsymbol{\lambda}^{u}$ and $\boldsymbol{\lambda}^{w}$ suggests that the net change in $\boldsymbol{\lambda}^{w}$ nearly satisfies the equation

$$
\frac{1}{r} \frac{\partial\left(r \boldsymbol{\lambda}^{u} \bar{\rho} \overline{\theta_{v}}\right)}{\partial r}+\frac{\partial\left(r \lambda^{w} \overline{\rho_{\theta}}\right)}{\partial z} \sim 0
$$

where $\theta_{v}$ denotes the virtual potential temperature and the overbars represent the basic fields in the forward run. This equation simply states that the perturbation of radial wind must be compensated by that of the vertical wind through the mass continuity equation when the contributions of the sound wave component are neglected. In a time-dependent model, this modulation is made through the terms associated with small time step.

Figures 6e and $6 \mathrm{f}$ show the balance of terms in the sensitivity fields with respect to potential temperature and the mixing ratio of water vapor at $1.41 \mathrm{~km}$. These figures indicate that buoyancy force and phase-change processes are responsible for the changes in $\boldsymbol{\lambda}^{\theta}$ and $\boldsymbol{\lambda}^{q}$. As for the buoyancy force, this reflects the fact that an increase (decrease) in potential temperature or mixing ratio of water vapor results in increasing (decreasing) vertical velocity. On the other hand, the phase-change processes reflects the fact that increase (decrease) of water vapor results in an increase (decrease) of potential temperature through the release of latent heat via condensation, while an increase (decrease) in potential temperature is unlikely (likely) to induce enhanced condensation. The changes in $\boldsymbol{\lambda}^{q_{\text {liq }}}$ are mainly due to the buoyancy force at $z=1 \mathrm{~km}$ (not shown).

The mechanisms responsible for the changes in the response function and in related processes are summarized in Fig. 7 in accordance with the term balances around $z=1 \mathrm{~km}$. This figure illustrates the process by which perturbation changes the tangential maximum velocity. For instance, consider the infinitesimal positive perturbation for the water vapor mixing ratio $\delta q$, where $\boldsymbol{\lambda}^{q}$ indicates strongly positive values, namely hurricane interior relative to the verification region. The perturbation intensifies the vertical velocity primarily through two terms. One involves the direct effect on the buoyancy force arising from changes in the weight of the

TABLE 1. List of abbreviations for the terms. An asterisk indicates terms used in the adjoint equation.

Terms in the forward equation and their corresponding terms in

Abbreviation the adjoint equation

\section{$\mathrm{ADV}, \mathrm{ADV}^{*} \quad$ Advective terms}

BUO, BUO* Buoyancy force term

COR, COR* Coriolis force term

DIF, DIF* Diffusion and viscosity term

MET, MET* Metric terms (including centrifugal force term)

SMA, SMA* Terms which are associated with the pressure derivation and integrated by small time step

PHA, PHA* Terms representing the phase change process RAD, RAD* Terms representing the radiation process 
(a)

(b)

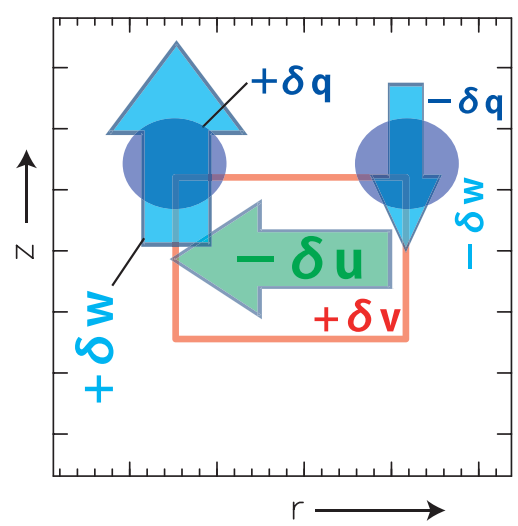

Time

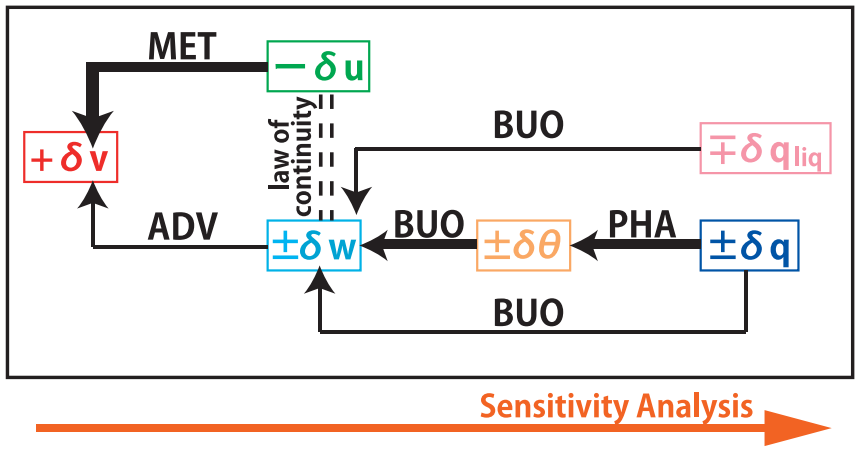

FIG. 7. (a) Schematic illustration of the process by which an infinitesimal perturbation yields a change in tangential velocity around $z=1 \mathrm{~km}$ within $4 \mathrm{~min}$. (b) Main terms associated with the changes in sensitivity fields. The thickness of the vector represents the relative importance of the process. Terms producing a quantitatively small contribution are omitted.

air parcel, and the other involves the potential temperature increase through phase change process from water vapor to liquid water. Subsequently, stronger radial inflow over the verification region takes place as a result of the compensation for enhanced vertical velocity. Such a radial flow change eventually leads to changes in tangential velocity through the metric term. The time scale of conversion from $\delta u$ to $\delta v$ will be discussed in section $6 \mathrm{a}$.

To assure the mechanism proposed here, we conduct another experiment by applying perturbations to the water vapor mixing ratio, $\delta q=+1 \mathrm{~g} \mathrm{~kg}^{-1}$, at one discretized time step. Note that $\boldsymbol{\lambda}^{q}$ takes high positive values in this experiment as suggested by Fig. 5 . The time evolution of the perturbations from $t=0$ to $t=$ 4 min by the nonlinear forward model is shown in Fig. 8 , which exhibits that the perturbation in $\delta q$ (denoted by gray shading in Fig. 8) contributes to an increase in the potential temperature and the evolution of the vertical and radial velocity changes. Figure $8 \mathrm{j}$ exhibits an increase of tangential velocity in the verification region $\left(+0.350 \mathrm{~m} \mathrm{~s}^{-1}\right.$ at a maximum $)$ and suggests that the proposed short-time-scale processes work as a response to the introduction of positive $\delta q$ in the nonlinear forward model.

\section{b. Sensitivity at $-20 \mathrm{~min}$}

Sensitivities at $-20 \mathrm{~min}$ for $r<80 \mathrm{~km}$ and $z<15 \mathrm{~km}$ are shown in Fig. 9. The figure exhibits high value regions in $\boldsymbol{\lambda}^{u}, \boldsymbol{\lambda}^{v}, \boldsymbol{\lambda}^{\theta}$, and $\boldsymbol{\lambda}^{q}$ downward to the surface according to the backward integration in time, which indicates that the changes in the surface momentum and sensible and latent heat fluxes can affect the tangential velocity in the verification region within this time scale.

This figure shows several notable features. 1) The sensitivities of $\boldsymbol{\lambda}^{u}$ and $\boldsymbol{\lambda}^{v}$ exhibit dipole patterns at the surface. The change of signs implies that the impact of the surface momentum exchange reverses at some radii. For example, a decrease of surface tangential velocity around $r=25 \mathrm{~km}$ weakens the tangential velocity in the verification region at $t=0 \mathrm{~min}$, while that around $r=$ $35 \mathrm{~km}$ intensifies. 2) The sensitivity fields with respect to $\boldsymbol{\lambda}^{\theta}$ and $\boldsymbol{\lambda}^{q}$ retain the dipole patterns, although the zero lines above the boundary layer are located away from the hurricane center (at $r=30 \mathrm{~km}$ ) in comparison with Figs. $5 \mathrm{e}$ and $5 \mathrm{f}$ and negative sensitivities are weak at the surface. The physical processes responsible for the latter features in $\boldsymbol{\lambda}^{\theta}$ and $\boldsymbol{\lambda}^{q}$ are the buoyancy terms and terms associated with the phase change in addition to the advection due to the boundary layer inflow (not shown). This implies that the oscillatory pattern of $\boldsymbol{\lambda}^{w}$ influences the values of $\boldsymbol{\lambda}^{\theta}$ and $\boldsymbol{\lambda}^{q}$.

To understand the dipole pattern in $\boldsymbol{\lambda}^{u}$ and $\boldsymbol{\lambda}^{v}$ as well as the oscillatory pattern in $\boldsymbol{\lambda}^{w}$, the sensitivity fields at intervals of $5 \mathrm{~min}$ are shown in Fig. 10. In this figure, the sign of the sensitivity field with respect to the wind fields varies rapidly in the eyewall region $(10<r<25 \mathrm{~km})$, while the change of sign is rather slow outside the eyewall region $(r>25 \mathrm{~km})$. A possible explanation for the rapid sign changes in $\boldsymbol{\lambda}^{u}$ and $\boldsymbol{\lambda}^{v}$ is given in section 6a.

The oscillatory pattern of $\boldsymbol{\lambda}^{w}$ is consistent with the values of $\boldsymbol{\lambda}^{u}$ through the mass continuity Eq. (13) if the contribution of sound waves is omitted. The positive 


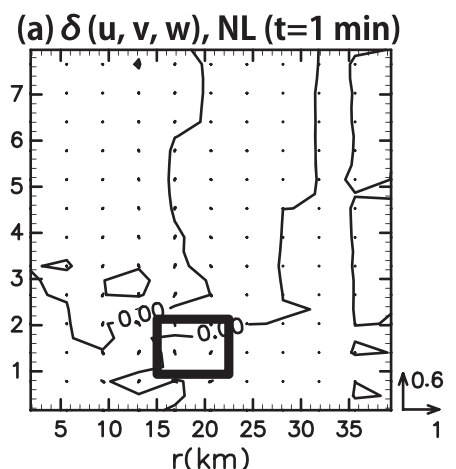

(d) $\delta(u, v, w), N L(t=2 \mathrm{~min})$

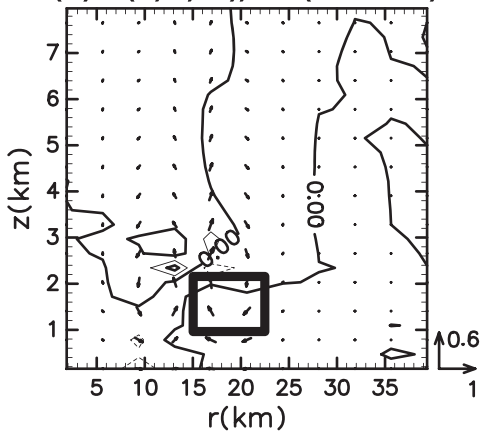

(g) $\delta(u, v, w), N L(t=3 \mathrm{~min})$

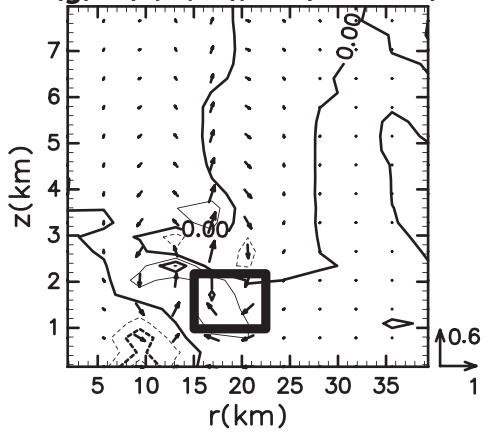

(j) $\delta(u, v, w), N L(t=4 \mathrm{~min})$

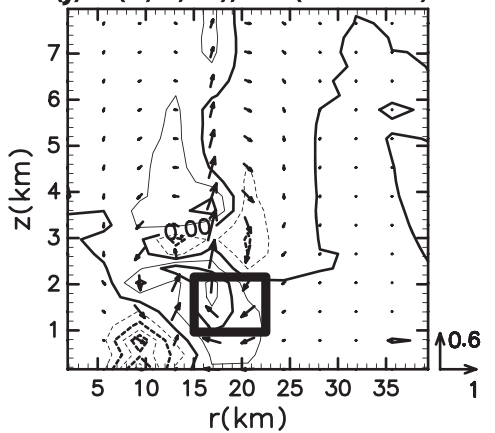

(b) $\delta \theta, \mathrm{NL}(\mathrm{t}=1 \mathrm{~min})$

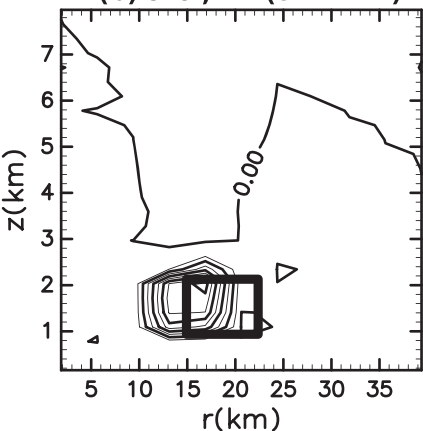

(e) $\delta \theta, \mathrm{NL}(\mathrm{t}=2 \mathrm{~min})$

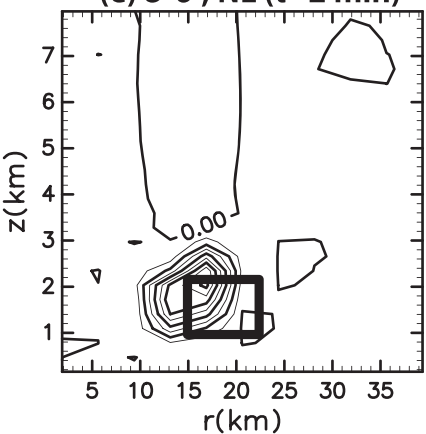

(h) $\delta \theta, \mathrm{NL}(\mathrm{t}=3 \mathrm{~min})$

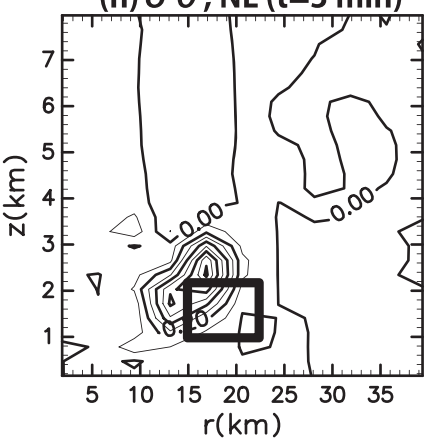

(k) $\delta \theta, \mathrm{NL}(\mathrm{t}=4 \mathrm{~min})$

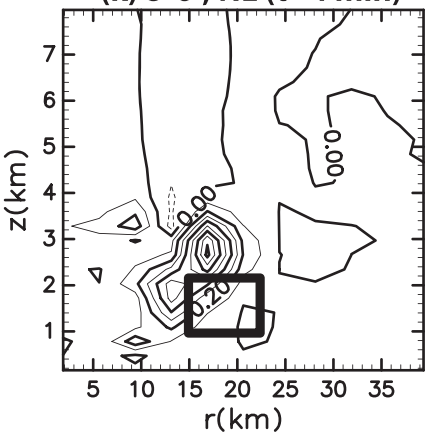

(c) $\delta \mathrm{q}, \mathrm{NL}(\mathrm{t}=1 \mathrm{~min})$

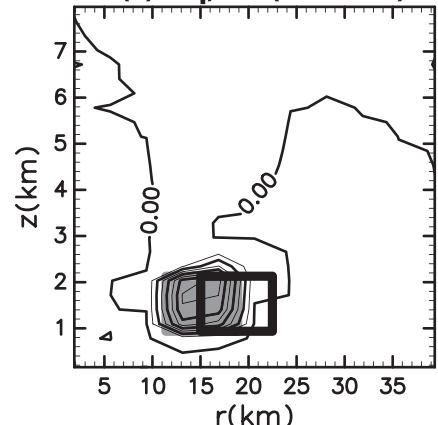

(f) $\delta \mathrm{q}, \mathrm{NL}(\mathrm{t}=2 \mathrm{~min})$

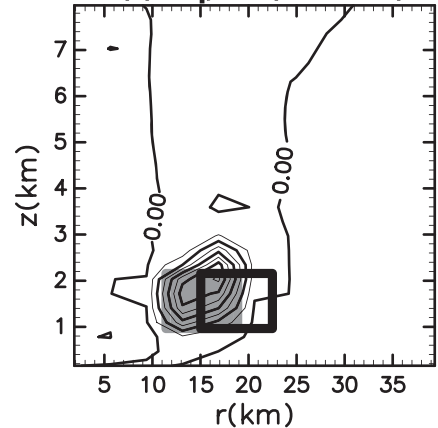

(i) $\delta \mathrm{q}, \mathrm{NL}(\mathrm{t}=3 \mathrm{~min})$

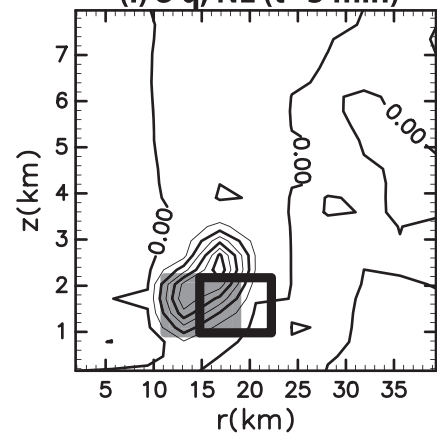

(I) $\delta \mathrm{q}, \mathrm{NL}(\mathrm{t}=4 \mathrm{~min})$

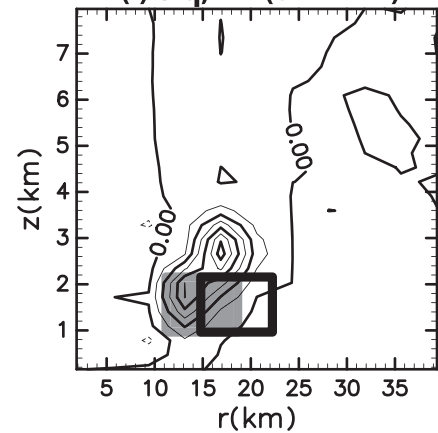

FIG. 8. Time evolution of positive $\delta q$ in the gray shaded area at $t=0$ min derived by the nonlinear forward model: (a)-(c) $t=1$, (d)-(f) $t=2$, (g)-(i) $t=3$, and (j)-(l) $t=4$ min. In all panels, black rectangles indicate the verification region and broken lines denote the negative values. In (a),(d), (g), (j) contours are $\delta v$ every $0.1 \mathrm{~m} \mathrm{~s}^{-1}$. Vectors superposed are $\delta u$ and $\delta w$; nit vectors are shown in the bottom of the rhs of each panel. In (b),(e),(h),(k) contours are $\delta \theta$ every $0.1 \mathrm{~K}$; in (c),(f),(i),(l) contours are $\delta q$ every $0.1 \mathrm{~g} \mathrm{~kg}^{-1}$. 
(a) Sensitivity: $\lambda_{u} @-20$ min.

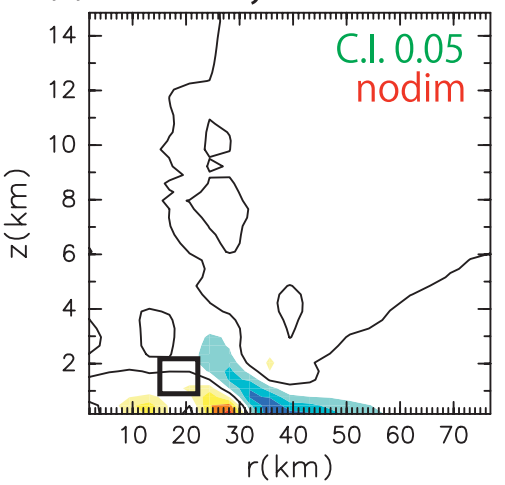

(c) Sensitivity: $\lambda_{w} @-20$ min.

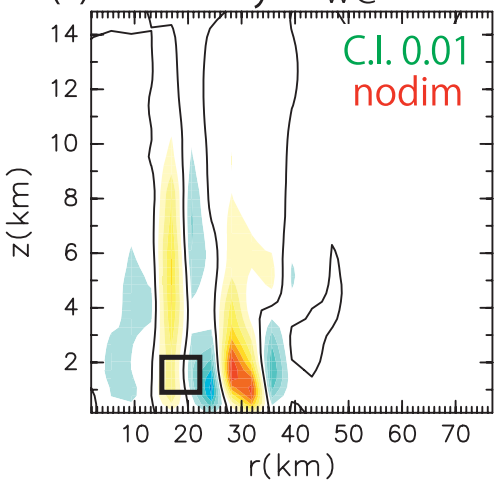

(e) Sensitivity: $\lambda_{\theta} @-20$ min.

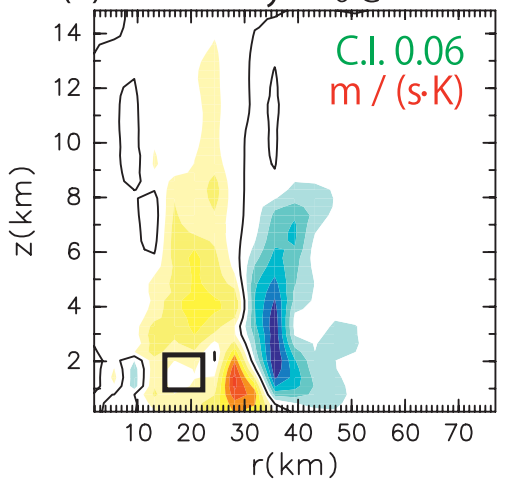

(b) Sensitivity: $\lambda_{\mathrm{v}} @-20 \mathrm{~min}$.

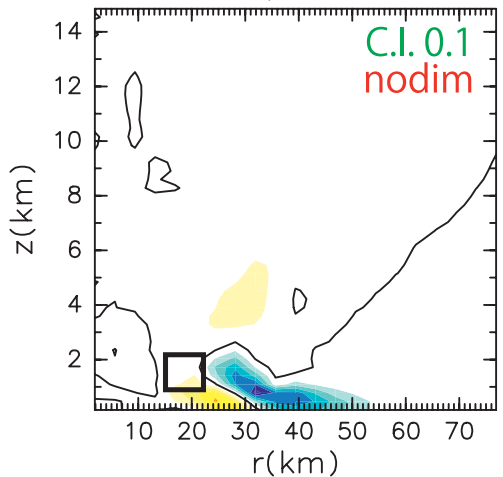

(d) Sensitivity: $\lambda_{\pi} @-20$ min.

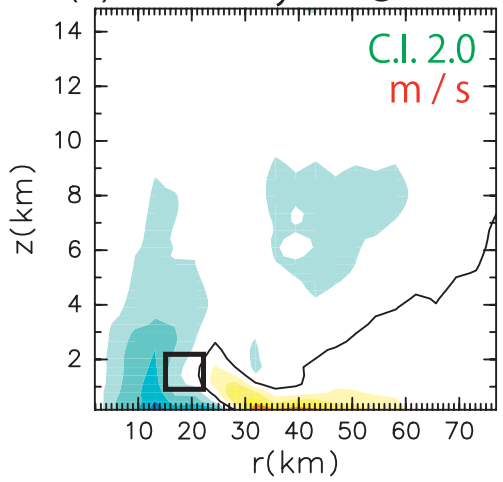

(f) Sensitivity: $\lambda_{\mathrm{a}} @-20 \mathrm{~min}$.

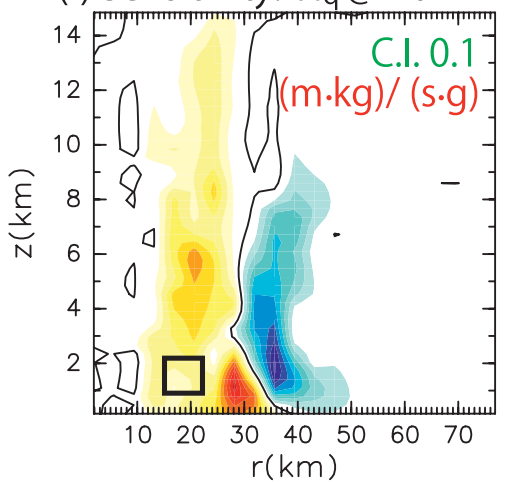

(g) Sensitivity: $\lambda_{\text {qliq }} @-20$ min.

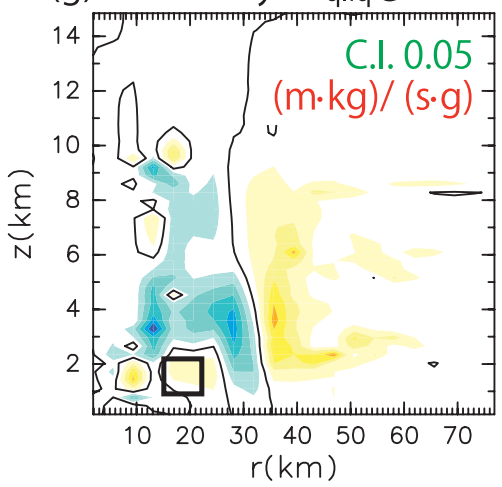

FIG. 9. As in Fig. 5, but at $-20 \mathrm{~min}$. 
(a) Sensitivity: $\lambda_{u} @-5 \min$.

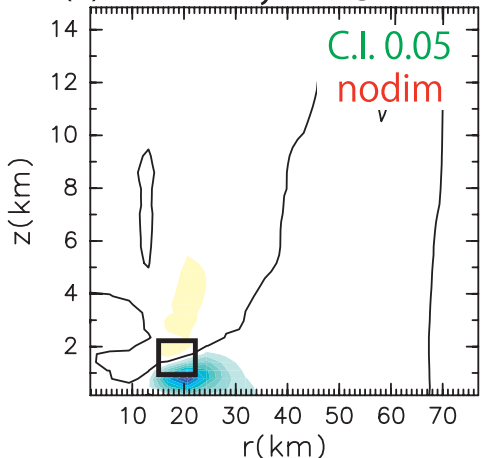

(d) Sensitivity: $\lambda_{\mathrm{u}} @-10 \mathrm{~min}$.

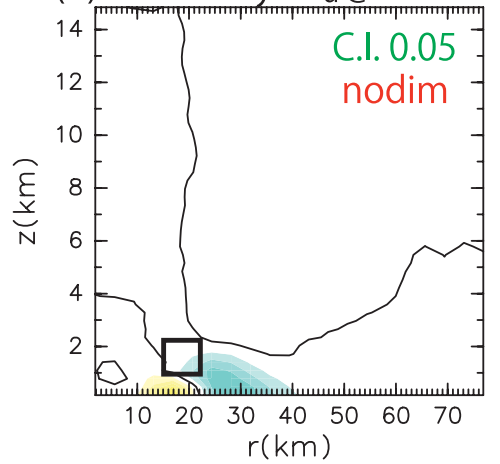

(g) Sensitivity: $\lambda_{u} @-15$ min.

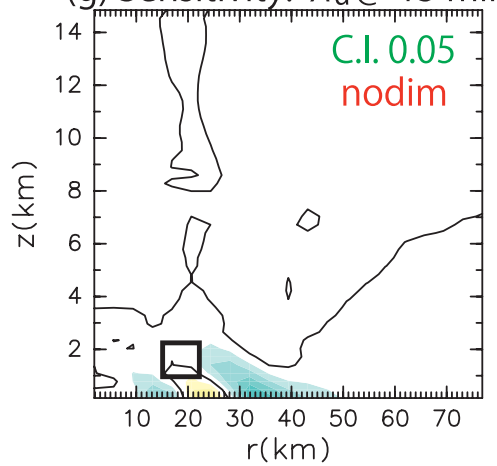

(j) Sensitivity: $\lambda_{u} @-20$ min.

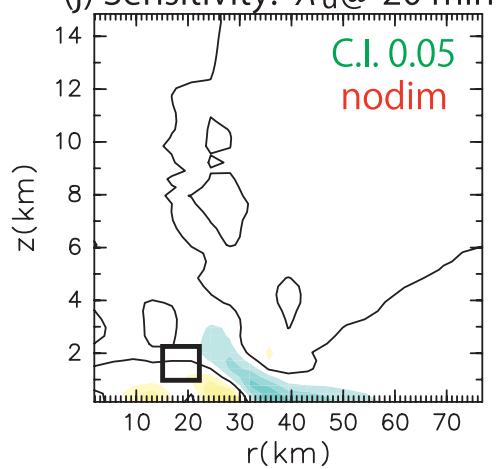

(b) Sensitivity: $\lambda_{v} @-5 \min$.

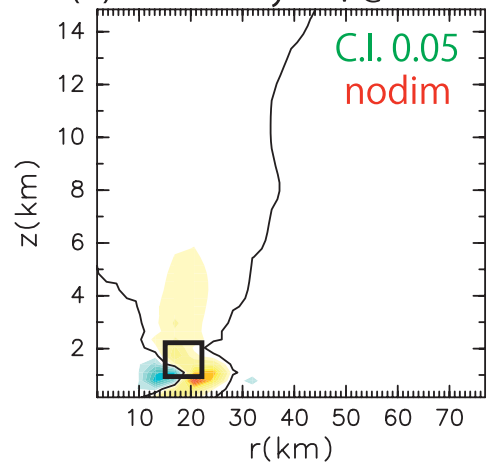

(e) Sensitivity: $\lambda_{\mathrm{v}} @-10 \mathrm{~min}$.

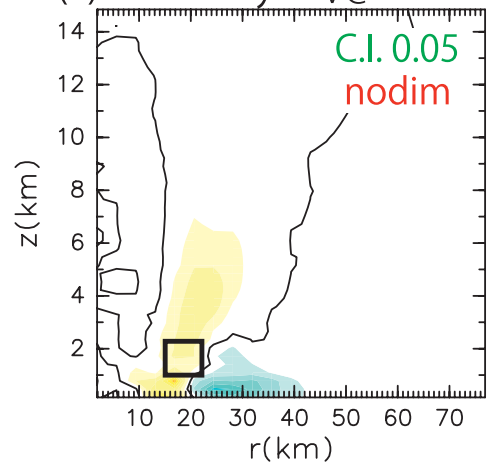

(h) Sensitivity: $\lambda_{v} @-15$ min.

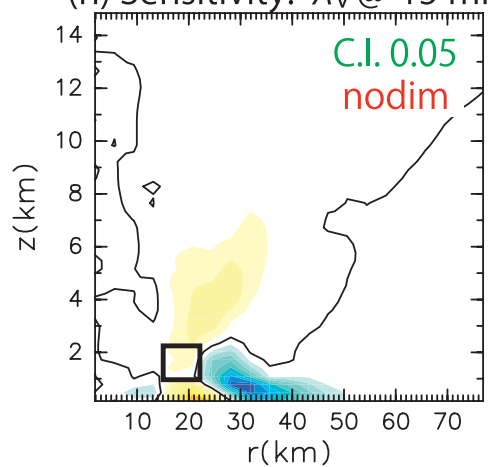

(k) Sensitivity: $\lambda_{\mathrm{v}} @-20 \mathrm{~min}$.

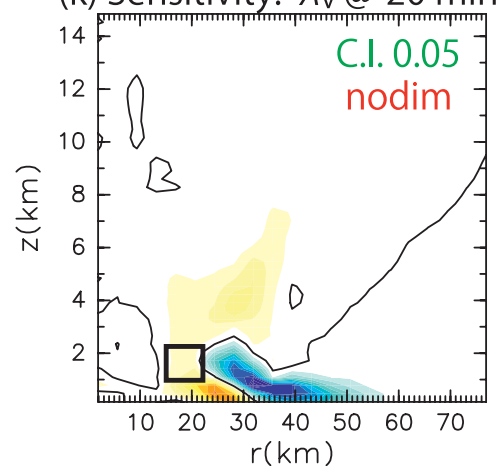

(c) Sensitivity: $\lambda_{w} @-5 \min$.

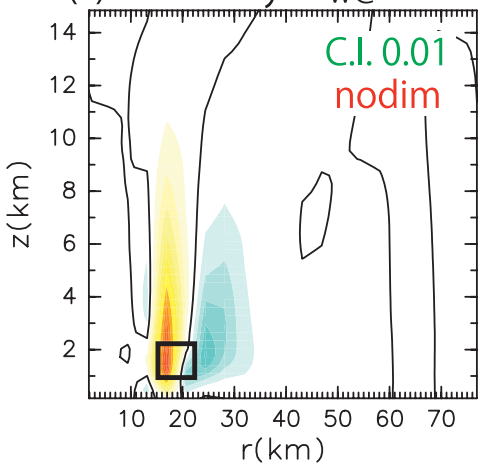

(f) Sensitivity: $\lambda_{w} @-10$ min.

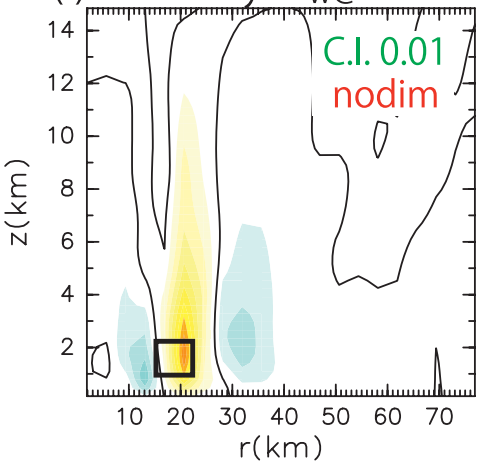

(i) Sensitivity: $\lambda_{w} @-15$ min.

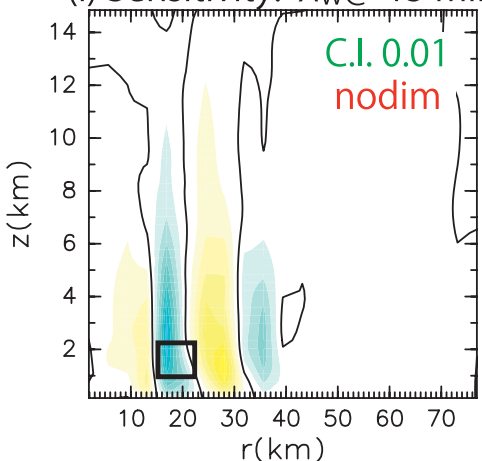

(I) Sensitivity: $\lambda_{w} @-20$ min.

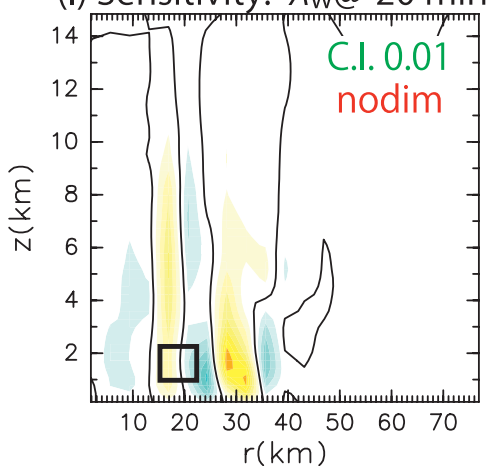

FIG. 10. As in Fig. 5, but with respect to the fields of $u\left(\mathrm{~m} \mathrm{~s}^{-1}\right), v\left(\mathrm{~m} \mathrm{~s}^{-1}\right)$, and $w\left(\mathrm{~m} \mathrm{~s}^{-1}\right)$ from -5 to $-20 \mathrm{~min}$. 
values of $\boldsymbol{\lambda}^{w}$ in the eyewall at the beginning of the backward integration (from 0 to $-10 \mathrm{~min}$ ) are sufficiently large. The behavior of $\boldsymbol{\lambda}^{w}$ explains why values of $\boldsymbol{\lambda}^{\theta}$ and $\boldsymbol{\lambda}^{q}$ are retained as positive quantities in the eyewall at $-20 \mathrm{~min}$ and the zero lines in $\boldsymbol{\lambda}^{\theta}$ and $\boldsymbol{\lambda}^{q}$ are shifted outward.

\section{c. Sensitivity at $-60 \mathrm{~min}$}

A 60 -min backward integration shows that the peaks of the sensitivity fields are farther away from the hurricane center and that the sensitivity fields are rather disturbed from the surface to the middle troposphere (Fig. 11). The sensitivity fields are affected by the boundary layer inflow and by short-lived updraft and downdraft motions in the low-middle troposphere outside the eyewall (Figs. 3b-d and $4 b-d$ ). It is noteworthy that the sensitivity fields of $\boldsymbol{\lambda}^{\theta}$ and $\boldsymbol{\lambda}^{q}$ still have positive values in most of the inner core region at the surface. Thus, the changes in the sensible and latent heat fluxes have relatively persistent effects in the tangential velocity of the verification region, while the effects of the momentum fluxes are limited to very short time scales.

\section{Sensitivity with respect to surface variables}

\section{a. Surface fluxes}

Sensitivities with respect to the surface momentum fluxes are shown in Fig. 12. It reveals that the response is relatively sensitive to $\boldsymbol{\tau}_{r z}$ and $\boldsymbol{\tau}_{\phi z}$ beneath the eyewall within $20 \mathrm{~min}$. In particular, it is shown that the influence of $\tau_{r z}$ is not large beyond $5 \mathrm{~min}$ ahead of the verification time. Another notable feature is that surface stress outside the eyewall acts on the response function in the opposite direction. The results are expected by the sensitivity with respect to wind fields in the previous section.

Sensitivities with respect to the sensible and latent surface fluxes are shown in Fig. 13. There are stronger positive values beneath the eyewall region and slight negative values in the exterior region of the hurricane during the period from -20 to $-5 \mathrm{~min}$ and from -40 to $-20 \mathrm{~min}$. It reflects the dipole pattern of $\boldsymbol{\lambda}^{\theta}$ and $\boldsymbol{\lambda}^{q}$ in the previous section since the positive perturbation of sensible and latent fluxes increases the potential temperature and mixing ratio of water vapor at the lowest layer. Thus, they finally result in the changes of the response function via the processes mentioned in the section 4 . This figure indicates that the magnitude of sensitivity with respect to the sensible and latent heat fluxes is almost same. It indicates that the changes in unit quantity of sensible and latent heat flux have nearly the same impact on the response function.
Figure 14 is a summary of the surface fluxes and state variables that intensify the tangential velocity in the verification region after $20 \mathrm{~min}$ of forward integration. As shown in this figure, the increases of momentum and heat flux have dual roles in the changes of the response function. This reflects the fact that negative values are seen outside the eyewall region in $\boldsymbol{\lambda}^{u}, \boldsymbol{\lambda}^{v}, \boldsymbol{\lambda}^{\theta}$, and $\boldsymbol{\lambda}^{q}$. This may be related partly to the results of recent studies ( $\mathrm{Xu}$ and Wang 2010; Miyamoto and Takemi 2010) in which the stronger maximum tangential wind is obtained by the elimination of surface entropy fluxes beyond some radii. However, more detailed discussion is left for future work because of several possible causes beyond the time scale concerned in the present study.

The peaks of the sensitivity with respect to the fluxes are shifted outward with further backward integration. Sensitivity fields with respect to radial surface stress are rather disturbed. This is due to the convective motion outside the eyewall region. A closer inspection reveals that the sensitivity with respect to heat fluxes has persistently positive values beneath the eyewall region, while the sensitivity with respect to the momentum fluxes beneath the eyewall is quantitatively small from -20 to $-60 \mathrm{~min}$. This corresponds to the fact that $\boldsymbol{\lambda}^{u}$ and $\lambda^{v}$ are shifted outside the eyewall beyond $-20 \mathrm{~min}$ whereas $\boldsymbol{\lambda}^{q}$ and $\boldsymbol{\lambda}^{\theta}$ have positive values in the eyewall (Figs. 11a,b,e,f).

Note that the changes in the response function depend not only on the sensitivity but also on the magnitude of the perturbations. We must keep in mind that the dissipation due to surface friction varies as the cube of the wind speed and the magnitude of latent heat flux is several times more than those of sensible heat fluxes in the present vortex.

\section{b. Sea surface temperature}

In the previous subsection, we show the sensitivities with respect to surface fluxes. They represent the firstorder change in $J$ due to the changes in fluxes per unit quantity. The response function is sensitive to an SST anomaly that affects the magnitudes of both sensible and latent heat fluxes. Therefore, it is worth calculating the sensitivity with respect to SST as one of the important oceanic variables. Figure 15 shows the sensitivity with respect to SST. During the period from -60 to $0 \mathrm{~min}$, the sensitivities are significantly positive beneath the eyewall and most of the neighboring region $(r<50 \mathrm{~km})$, while there exists a negative sensitivity. In particular, the highly positive sensitivity with respect to SST appears beneath the eyewall. This fact reflects that the magnitude of heat-flux perturbation depends on the wind speed. 


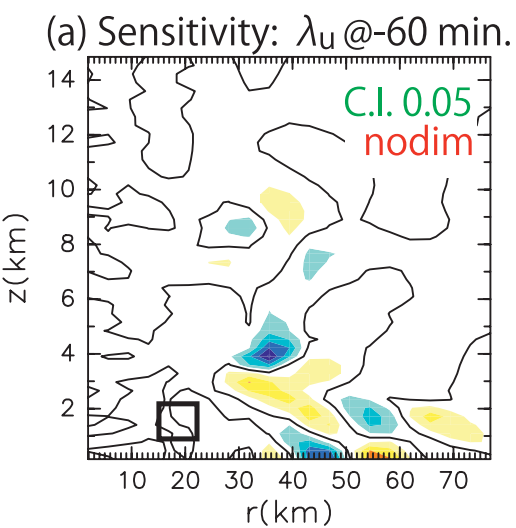

(c) Sensitivity: $\lambda_{w} @-60$ min.

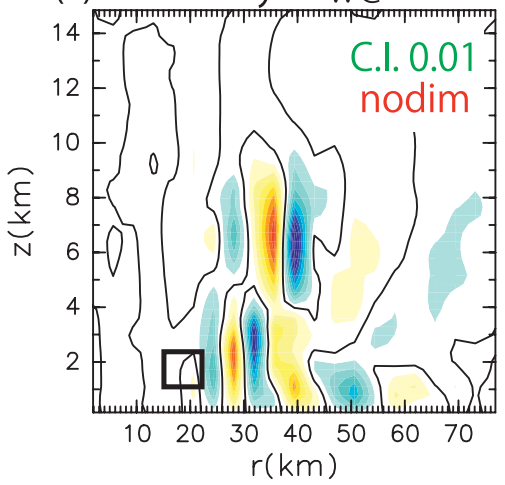

(e) Sensitivity: $\lambda_{\theta} @-60$ min.

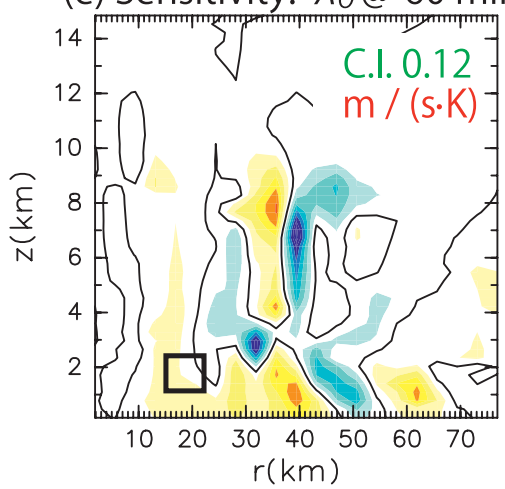

(b) Sensitivity: $\lambda_{\mathrm{v}} @-60 \mathrm{~min}$.

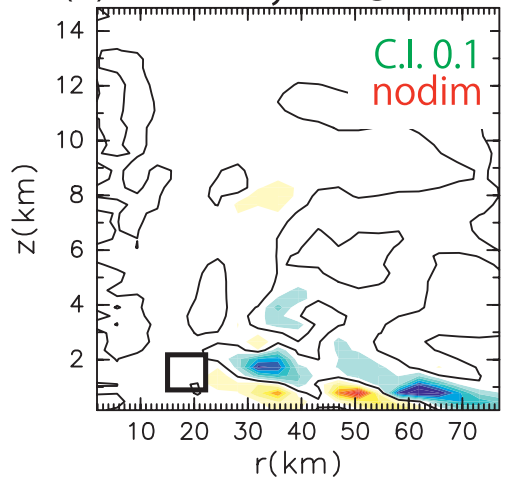

(d) Sensitivity: $\lambda_{\pi} @-60$ min.

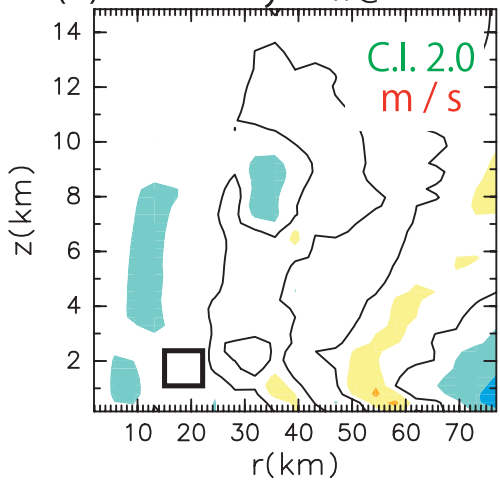

(f) Sensitivity: $\lambda_{\mathrm{g}} @-60 \mathrm{~min}$.

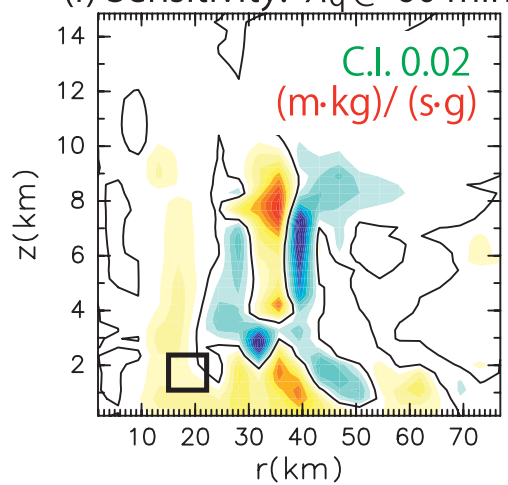

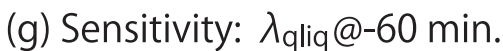

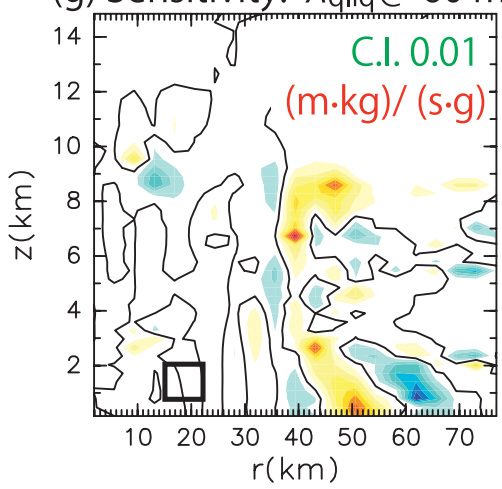

FIG. 11. As in Fig. 5, but at $-60 \mathrm{~min}$. 
$(\times .01)$ (a) $\lambda^{\operatorname{Trz}}$

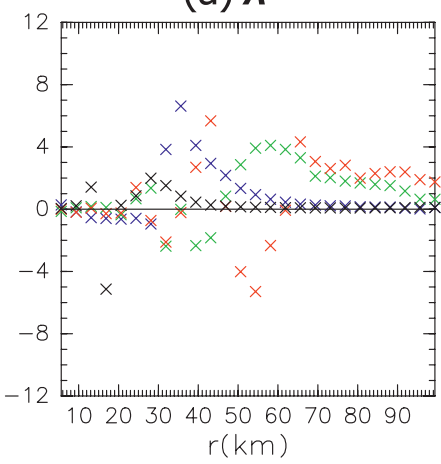

$(\times .01)$ (b) $\lambda^{\tau \phi z}$

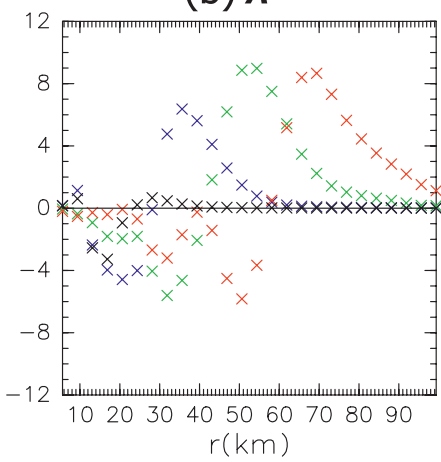

$x-5 \min <t<0 \min \quad x-20 \min <t<-5 \min$

$-40 \min <t<-20 \min$

$-60 \min <t<-40 \min$

FIG. 12. The sensitivity of $J$ with respect to (a) $\tau_{r z}$ and (b) $\tau_{\phi z}$ at each grid point. The sensitivity corresponds to the changes of fluxes per minute per unit quantity between -5 and 0 min (black), between -20 and -5 min (blue), between -40 and -20 min (green), and between -60 and -40 min (red).

Since the sensitivity is defined as $\partial J / \partial \mathbf{c}$, the first-order change in the response function $\delta J$ at the verification time is obtained from multiplying the sensitivity by the magnitude of perturbation-that is, $\delta J=(\partial J / \partial \mathbf{c}) \cdot \delta \mathbf{c}$. If a positive SST perturbation by $1 \mathrm{~K}$ is applied in the region from $r=10$ to $r=50 \mathrm{~km}$ for $1 \mathrm{~h}$, the amount is equivalent to first-order changes in $J$ of $24.4 \mathrm{~m} \mathrm{~s}^{-1}$ (on the grid-averaged value of $3.05 \mathrm{~m} \mathrm{~s}^{-1}$ in the verification region). The rapid intensification from Saffir-Simpson scale category 1 (34-43 $\left.\mathrm{m} \mathrm{s}^{-1}\right)$ to category $5\left(>71 \mathrm{~m} \mathrm{~s}^{-1}\right)$ can occur within a day over the warm ocean eddies (Lin et al. 2005, 2008). The rates obtained in this study account, in part, for the rapid intensification of hurricanes, although the time scales considered in this study are limited up to $60 \mathrm{~min}$.

It should be noted, however, that the hurricane intensification is, in reality, related to the upper-ocean heat content rather than the SST itself since hurricane passage alters the SST through the turbulent mixing and Ekman pumping in the upper ocean (Price 1981; Price et al. 1994; Scharroo et al. 2005; Lin et al. 2005; Wu et al. 2007b). This evaluation should be regarded as a potential impact of upper-ocean warm (cold) eddies on suppression (enhancement) of the SST decrease due to the hurricane passage.

\section{Discussion}

\section{a. Perturbations in radial and tangential velocity}

In section 4 , the sensitivities of $\boldsymbol{\lambda}^{u}$ and $\boldsymbol{\lambda}^{v}$ affect each other primarily through the metric terms (Figs. 6a,b), and the signs of the sensitivity field with respect to the wind fields seem to be oscillatory (Fig. 10). They occur
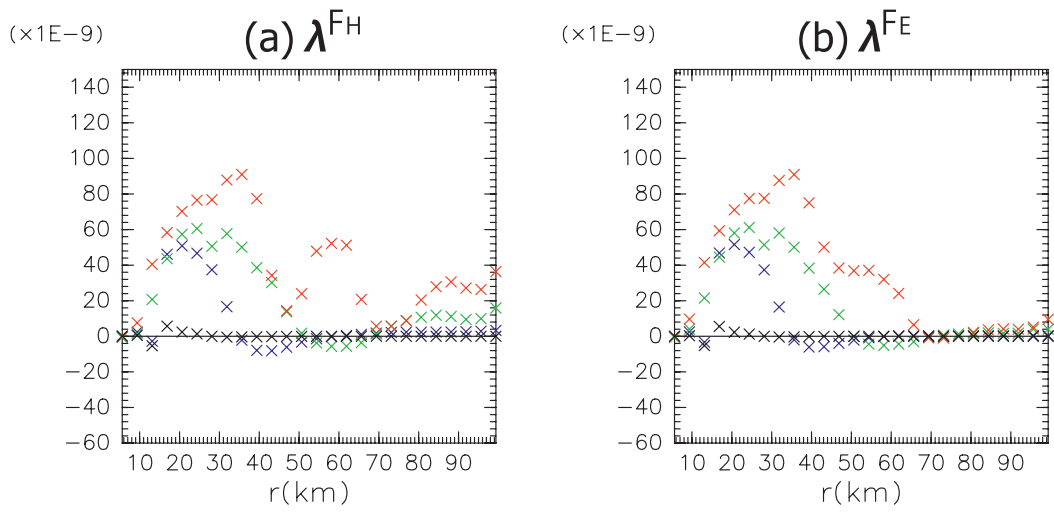

$x-5 \min <t<0 \min \quad x-20 \min <t<-5 \min$

FIG. 13. As in Fig. 12, but for the sensitivity of $J$ with respect to (a) sensible and (b) latent heat fluxes at each grid point. 
(a) Momentum Fluxes

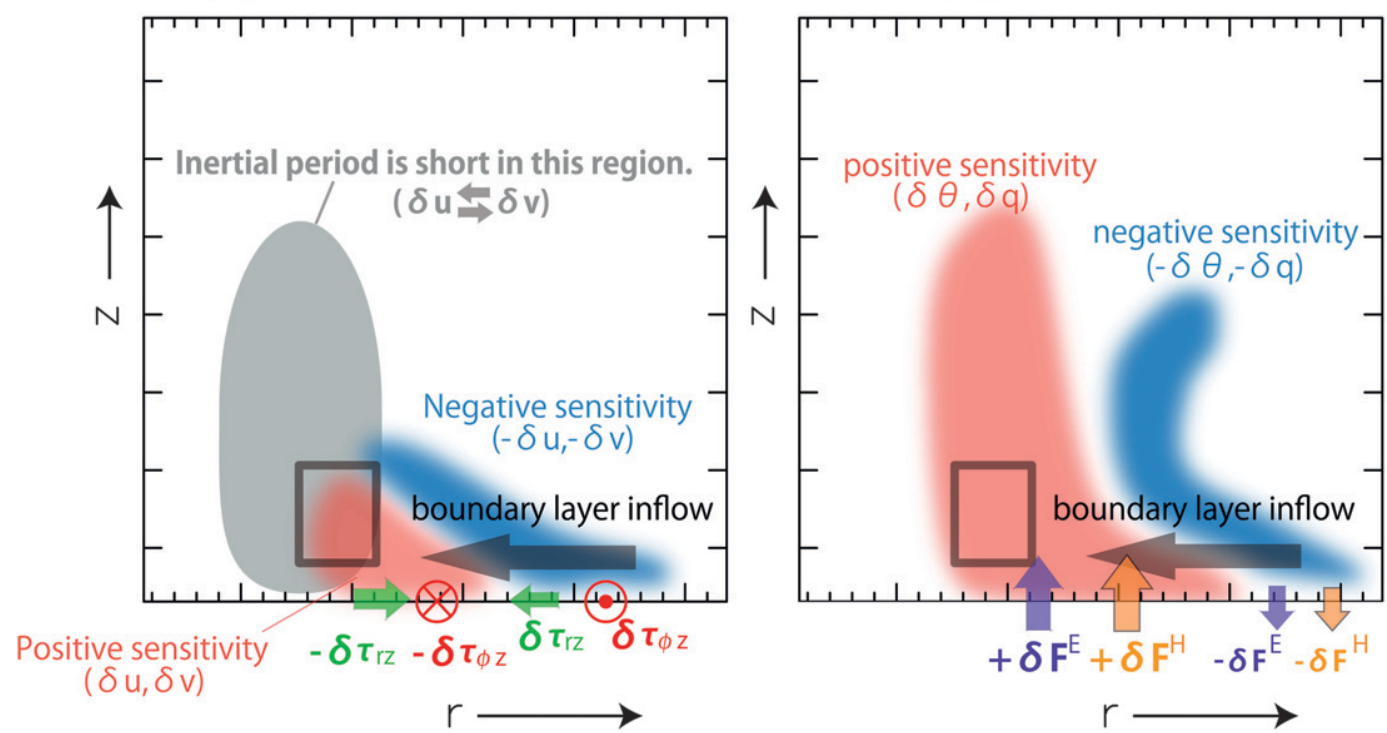

FIG. 14. Summary of the perturbations in surface fluxes and state variables that intensify the maximum tangential velocity after $20 \mathrm{~min}$ of forward integration: (a) surface stresses and (b) heat fluxes. The rectangle indicates the verification region. The gray shaded area in (a) indicates the region where the local inertial period is short (see section 6a for detail) rapidly in the eyewall region $(10<r<25 \mathrm{~km})$, whereas they are rather slow outside the eyewall $(r>25 \mathrm{~km})$. We would like to provide an explanation for these changes in this subsection.

Consider the perturbations of horizontal velocity added to the reference fields,

$$
u=\langle u\rangle+\delta u \text { and } v=\langle v\rangle+\delta v,
$$

where the angle brackets represent the values in the reference field. Neglecting the perturbation of the pressure fields, the radial and tangential equations of motion yield

$$
\frac{d_{\mathrm{ref}}}{d t}\left(\begin{array}{l}
\delta u \\
\delta v
\end{array}\right)=\left(\begin{array}{cc}
-\frac{\partial\langle u\rangle}{\partial r} & f+\frac{2\langle v\rangle}{r} \\
-f-\frac{\langle v\rangle}{r}-\frac{\partial\langle v\rangle}{\partial r} & -\frac{\langle u\rangle}{r}
\end{array}\right)\left(\begin{array}{l}
\delta u \\
\delta v
\end{array}\right) .
$$

Here, diffusion terms are omitted. The operator $d_{\mathrm{ref}} / d t$ represents

$$
\frac{d_{\mathrm{ref}}}{d t} \equiv \frac{\partial}{\partial t}+\langle u\rangle \frac{\partial}{\partial r}+\langle w\rangle \frac{\partial}{\partial z} .
$$

Considering the magnitude of the terms, the perturbation of horizontal motion is shown to be oscillatory in the eyewall region. Elementary calculus yields the period of local inertial oscillation of perturbations $2 \pi / I$, where

$$
I^{2} \equiv\left(f+\frac{\langle v\rangle}{r}+\frac{\langle v\rangle}{\partial r}\right)\left(f+\frac{2\langle v\rangle}{r}\right)-\frac{1}{2}\left(\frac{\partial\langle u\rangle}{\partial r}-\frac{\langle u\rangle}{r}\right)^{2} .
$$

If we neglect the radial velocity of reference field, $I$ is simply the local inertial frequency in the gradientbalanced vortex (e.g., Willoughby 1988). The time-mean field (from -60 to $0 \mathrm{~min}$ ) of $2 \pi / I$ is shown in Fig. 16. The time scale of the sign change in the perturbation of

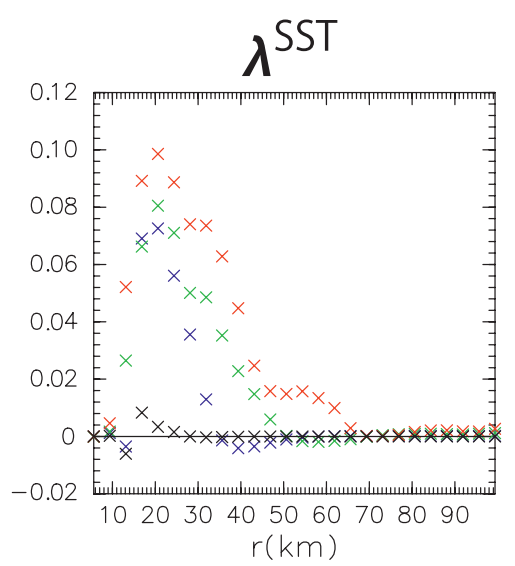

$$
\begin{array}{ll}
x-5 \min <\mathrm{t}<0 \min & x-20 \min <\mathrm{t}<-5 \min \\
x-40 \min <\mathrm{t}<-20 \min & \times-60 \min <\mathrm{t}<-40 \min
\end{array}
$$

FIG. 15. As in Fig. 12, but for the sensitivity with respect to SST. 


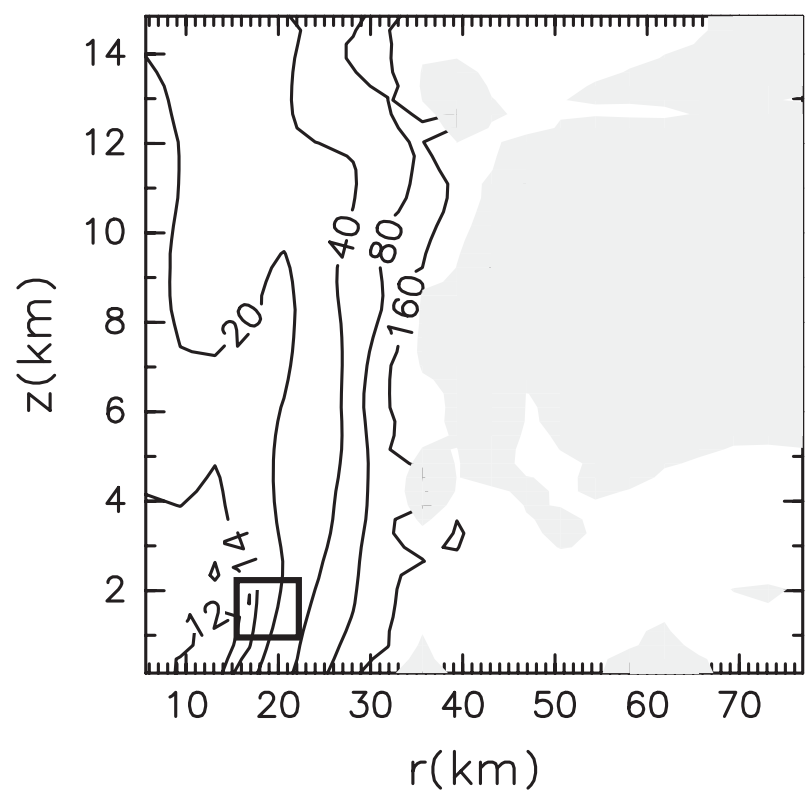

FIG. 16. Local inertial period. Contour values are 12, 14, 20, 40, 80 , and $160 \mathrm{~min}$. Regions in the inertially unstable condition are shaded. The rectangle indicates the verification region.

horizontal motion is half of this period, that is, a few minutes to $20 \mathrm{~min}$ in the eyewall region, while the time scale is rather long outside the eyewall. It is consistent with the sign changes of $\boldsymbol{\lambda}^{u}$ and $\boldsymbol{\lambda}^{v}$ in the eyewall and it also explains that the sign changes are quite slow outside the eyewall.

Thus, the sensitivities of $\boldsymbol{\lambda}^{u}$ and $\boldsymbol{\lambda}^{v}$ interchange rapidly in the eyewall. The oscillatory pattern of $\boldsymbol{\lambda}^{w}$ can be explained by the values of $\boldsymbol{\lambda}^{u}$ through the mass continuity
Eq. (13) if the contribution of sound waves is omitted. It should be noted that the analysis here is valid just after introducing the perturbation of horizontal motion since the time evolution of pressure fields is neglected in this analysis. In that sense, the period shown in Fig. 16 is locally legitimate.

\section{b. Importance of uncertainty in air-sea exchange coefficients}

If we take air-sea exchange coefficients $\left(C_{D}, C_{H}\right.$, and $C_{E}$ ) as the independent variables instead of surface stress and heat fluxes, the importance of each uncertainty in air-sea exchange coefficients can be measured. The sensitivity with respect to air-sea exchange coefficients will be calculated in this subsection. This information is useful, for instance, in the practical application aimed at specifying the air-sea exchange coefficients from the flight-level observations in the eyewall region as proposed by Ito et al. (2010).

Figure 17 shows the sensitivity of $J$ with respect to $C_{D}, C_{H}$, and $C_{E}$. Blue marks indicate the sensitivity corresponding to the changes in the coefficients during the period from -20 to 0 min, green marks during the period from -40 to $0 \mathrm{~min}$, and red marks during the period from -60 to $0 \mathrm{~min}$. These sensitivities are related to the sensitivity fields with respect to the state variables. For example, dipole patterns of $\boldsymbol{\lambda}^{q}$ correspond to $\boldsymbol{\lambda}^{C_{E}}$ since positive perturbations of $C_{E}$ continue to fuel more humid air at the surface.

Figure 17a indicates that the maximum tangential velocity is sensitive to $C_{D}$ values. The response function decrease as a result of enlarging $C_{D}$ from $r=10$ to $r=40 \mathrm{~km}$, while it increases as a result of enlarging $C_{D}$ beyond $r=40 \mathrm{~km}$. (a)

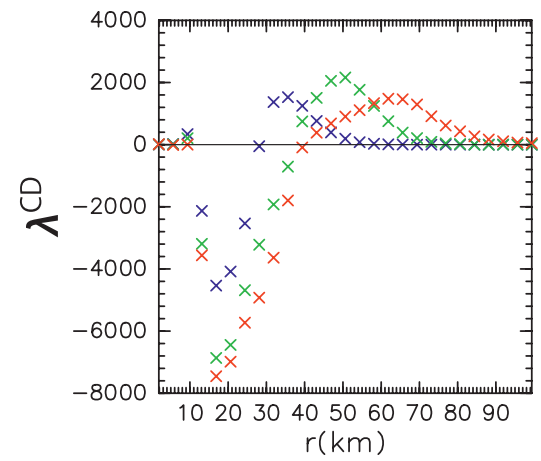

(b)

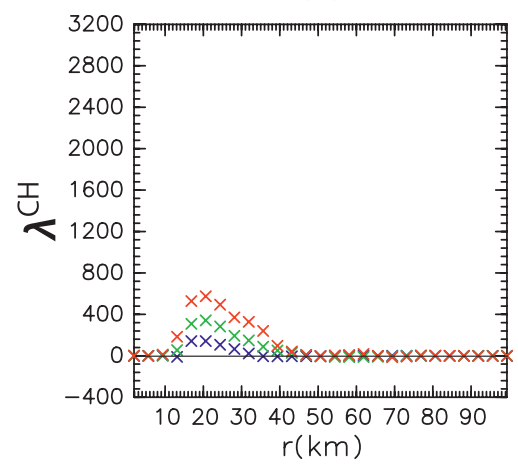

(c)

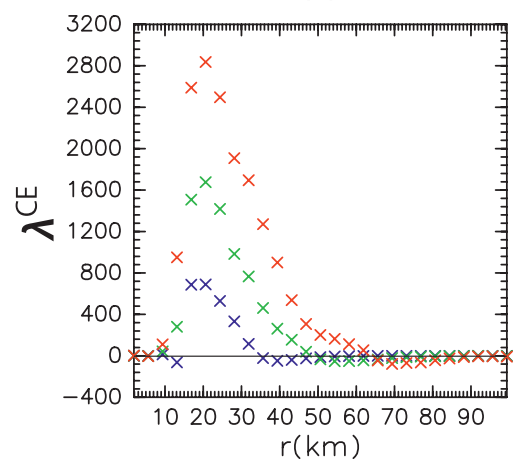

$$
x-20 \min <t<0 \min \quad x-40 \min <t<0 \min \quad x-60 \min <t<0 \min
$$

FIG. 17. The sensitivity of $J$ with respect to the values of (a) the drag coefficient, (b) the sensible heat exchange coefficient, and (c) the latent heat exchange coefficient at each grid point. The sensitivities corresponding to changes between -20 and 0 min (blue), between -40 and 0 min (green), and between -60 and 0 min (red) are as shown. 
(a) "CDOut+1.0" minus "Reference"

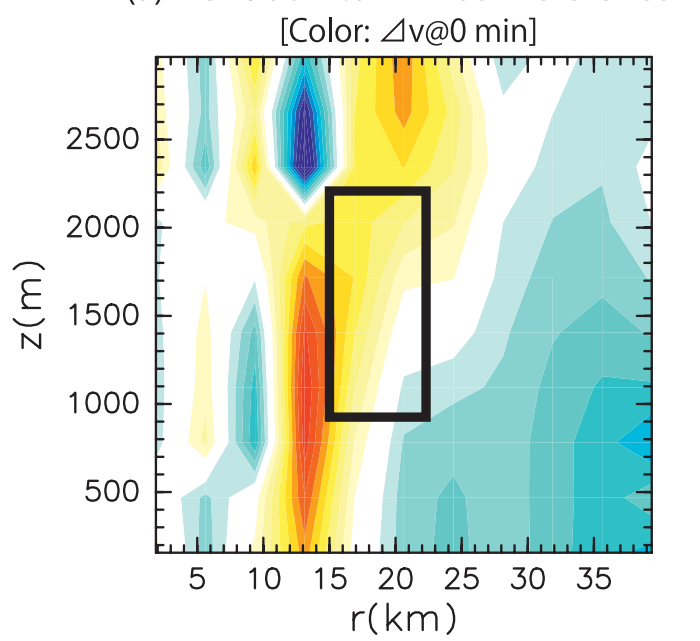

(b) "CDOut+0.5" minus "Reference"

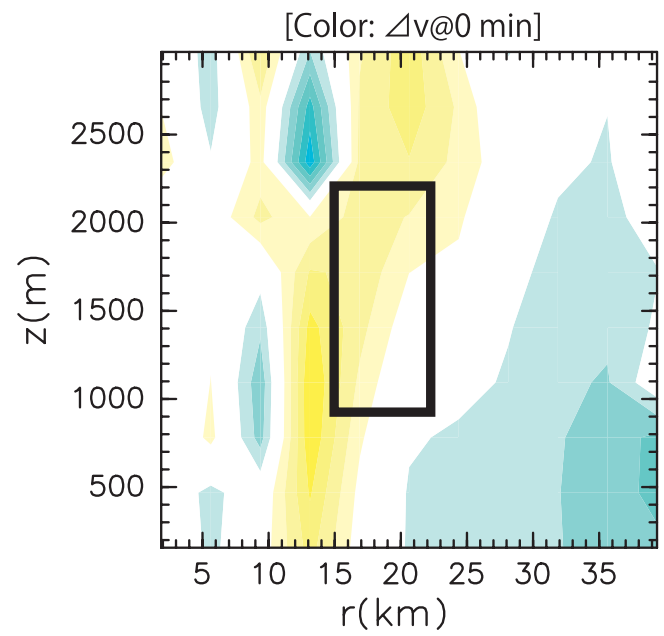

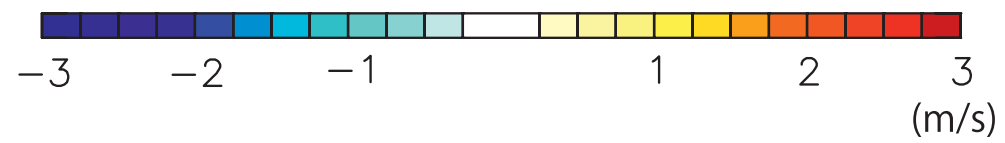

FIG. 18. Differences of tangential velocity between (a) " $C_{D}$ Out +1.0 " and "Reference" and between (b) " $C_{D}$ Out + 0.5 " and "Reference." The rectangle in each panel indicates the verification region.

To our knowledge, the latter aspect has not been pointed out by previous studies. We further conduct two experiments in which a finite positive perturbation of $0.5 \times 10^{-3}$ or $1.0 \times 10^{-3}$ is added to the original $C_{D}$ from $r=45$ to $r=$ $82.5 \mathrm{~km}$ to confirm this fact. These experiments are named " $C_{D}$ Out +0.5 " and " $C_{D}$ Out +1.0 ," respectively. After the forward integration of $60 \mathrm{~min}, J$ is increased by 3.07 and $4.96 \mathrm{~m} \mathrm{~s}^{-1}$, respectively (Fig. 18). This result supports our finding that stronger friction in the hurricane exterior can slightly intensify the tangential velocity.

If we simply multiply by $1.0 \times 10^{-3}$, which is a typical scale for the discrepancies among air-sea momentum exchange coefficients proposed recently for extreme conditions (Powell et al. 2003; Donelan et al. 2004; Black et al. 2007; Moon et al. 2007), the maximum first-order impact on $J$ after the forward integration of $60 \mathrm{~min}$ is $-7.45 \mathrm{~m} \mathrm{~s}^{-1}$ (or a grid-averaged value of $-0.93 \mathrm{~m}$ $\mathrm{s}^{-1}$ in the verification region) by a single gridpoint perturbation of $C_{D}$ at $r=16.875 \mathrm{~km}$ and $-32.08 \mathrm{~m}$ $\mathrm{s}^{-1}$ (or a grid-averaged value of $-4.01 \mathrm{~m} \mathrm{~s}^{-1}$ in the verification region) by perturbations from $r=10$ to $r=$ $40 \mathrm{~km}$. This calculation indicates that uncertainty in the air-sea exchange coefficients beneath the eyewall regions is important in terms of the maximum tangential velocity, even for the short time scale.

The sensitivity with respect to sensible and latent heat exchange coefficient is shown in Figs. $17 \mathrm{~b}$ and 17c, respectively. The sensitivity with respect to the sensible heat exchange coefficients $\boldsymbol{\lambda}^{C_{H}}$ is quite small compared to the sensitivities with respect to the other coefficients. The magnitude of the sensitivity beneath the eyewall is far larger than that outside the eyewall since the sensitivity fields with respect to these coefficients also depend on the wind speed. The values for $\boldsymbol{\lambda}^{C_{H}}$ and $\boldsymbol{\lambda}^{C_{E}}$ are positive in almost all areas, although slight negative values are seen in the hurricane exterior. This fact indicates that an increase (decrease) of the entropy generally yields stronger (weaker) tangential velocity, while an increase (decrease) in hurricane exterior has a weak effect in an opposite sense, which is also consistent with results reported in this study.

These results support that the improvement of $C_{D}$ and $C_{E}$ under the high-wind condition can contribute to the better hurricane intensity prediction and optimal estimate (Moon et al. 2007; Bender et al. 2007; Ito et al. 2010). It is worth mentioning that $J$ is not sensitive to changes in the air-sea exchange coefficients beneath the eye, at least in this time scale. This supports the work of Bryan and Rotunno (2009a), which showed that the high-entropy air in the eye does not qualitatively affect the maximum tangential velocity.

\section{Summary}

To understand the role of sea surface fluctuations that impact hurricane intensity in the mature stage, an adjointbased sensitivity analysis has been performed by backward integration with a cloud-permitting nonhydrostatic 
axisymmetric adjoint model. In our study, we have focused on a period covering some $60 \mathrm{~min}$, since this is the period of reasonable linearization for small perturbations. The tangential velocity in the eyewall region at the top of the boundary layer is taken as the response function $J$.

A 4-min backward integration shows that the sensitivities with respect to the radial velocity $\boldsymbol{\lambda}^{u}$ are negative around the verification region, which indicates that adding a negative perturbation of radial velocity to the reference field (stronger inflow) strengthens the maximum tangential velocity. The sensitivities with respect to the vertical velocity $\boldsymbol{\lambda}^{w}$, potential temperature $\boldsymbol{\lambda}^{\theta}$, and mixing ratio of water vapor $\boldsymbol{\lambda}^{q}$ show dipole patterns centered on the verification region. The processes associated with these sensitivities are analyzed by means of the term balance of the adjoint equations. The results show that the changes in $\boldsymbol{\lambda}^{u}$ are mainly due to the metric term. A dipole pattern for $\boldsymbol{\lambda}^{w}$ can be basically explained through the mass continuity equation. The changes in $\boldsymbol{\lambda}^{\theta}$ and $\boldsymbol{\lambda}^{q}$ are mainly explained by buoyancy force terms and terms associated with the condensation. This suggests that changes in water vapor mixing ratio affect the tangential velocity in highly localized zones around the verification region and that there is therefore no need to call for additional changes in the central pressure field.

As a result of the 20-min backward integration, the peaks of the sensitivity fields are shifted to the surface. This indicates that the changes at the surface can affect the tangential velocity in the verification region within this time scale. The shift of the peaks is influenced by the boundary layer inflow in the reference field. In addition, the shift of the zero line in $\boldsymbol{\lambda}^{\theta}$ and $\boldsymbol{\lambda}^{q}$ follows the changes in $\boldsymbol{\lambda}^{w}$. The sensitivity fields with respect to wind fields seem oscillatory within this period, which can be explained by the local inertial motion. Beyond the 20-min backward integration, the sensitivity fields become highly disturbed and range from the surface to the middle troposphere and are located farther from the center. This is due to the active vertical motion caused by the convective updraft and downdraft outside the eyewall region, in addition to the boundary layer inflow.

The sensitivities with respect to the air-sea fluxes and SST are consistent with the changes in the state variables. Increases in momentum and heat fluxes play dual roles in instigating changes of the response function. This dual role of surface fluxes may be relevant to results reported in recent studies (Xu and Wang 2010; Miyamoto and Takemi 2010).

Of course, care should be taken for the sensitivity obtained in this simplified model physics. This study is just related to the physics included in the axisymmetric numerical model. The process associated with the vortex Rossby waves and spiral rainbands are not included.
Furthermore, the time scale of physical processes is limited up to $60 \mathrm{~min}$. Future work will be directed to a three-dimensional model and the extended sensitivity analysis, which may be valid for longer time scale (e.g., Torn and Hakim 2008). Nevertheless, we believe that this study contributes to the enhanced understanding of the time-dependent dynamic nature in a mature hurricane.

Acknowledgments. We especially thank Dr. K. Emanuel, who generously provided the forward model, and Dr. T. Takemi, Dr. M. Sawada, and Mr. K. Nishina for providing useful comments. This work was partly supported by a Grant-in-Aid from Green Innovation of Universal Marine Industry (UMI) and the Japan Society for the Promotion of Science (JSPS) Fellows. The figures were produced by the GFD DENNOU Library. In this research work we used the supercomputer of ACCMS, Kyoto University.

\section{APPENDIX}

\section{Forward Model and Adjoint Model Used in the Present Study}

The governing equations used in the forward model and adjoint model are summarized in this appendix. Note that the equations are written in a continuous form for simplicity, although forward and backward model integrations are done with a discretized form. A more detailed description of the forward model is given in Rotunno and Emanuel (1987).

Neglecting diffusion terms and dumping terms in the sponge layer, the equations used in the forward model are as follows:

$$
\frac{\partial u}{\partial t}=\underbrace{-u \frac{\partial u}{\partial r}-w \frac{\partial u}{\partial z}}_{\operatorname{ADV}(u, w)} \underbrace{-f v}_{\operatorname{COR}(v)} \underbrace{-\frac{v^{2}}{r}}_{\operatorname{MET}(v)}+\operatorname{SMA}(u, w, \pi),
$$

$$
\frac{\partial v}{\partial t}=\underbrace{-u \frac{\partial v}{\partial r}-w \frac{\partial v}{\partial z}}_{\mathrm{ADV}(u, v, w)}+\underbrace{f u}_{\operatorname{COR}(u)}+\underbrace{\frac{u v}{r}}_{\operatorname{MET}(u, v)}
$$

$$
\begin{aligned}
\frac{\partial w}{\partial t}= & \underbrace{-u \frac{\partial w}{\partial r}-w \frac{\partial w}{\partial z}}_{\operatorname{ADV}(u, w)}+\underbrace{g\left\{\frac{\theta-\bar{\theta}}{\bar{\theta}}+0.61(q-\bar{q})-q_{\mathrm{liq}}\right\}}_{\operatorname{BUO}\left(\theta, q, q_{\mathrm{liq}}\right)} \\
& +\operatorname{SMA}(u, w, \pi), \\
\frac{\partial \pi}{\partial t}= & \operatorname{SMA}(u, w, \pi),
\end{aligned}
$$




$$
\begin{aligned}
& \frac{\partial \theta}{\partial t}=\underbrace{-u \frac{\partial \theta}{\partial r}-w \frac{\partial \theta}{\partial z}}_{\operatorname{ADV}(u, w, \theta)}+\operatorname{PHA}\left(q, q_{\mathrm{liq}}\right)+\operatorname{RAD}(\theta), \\
& \frac{\partial q}{\partial t}=\underbrace{-u \frac{\partial q}{\partial r}-w \frac{\partial q}{\partial z}}_{\operatorname{ADV}(u, w, q)}+\operatorname{PHA}\left(\theta, q, q_{\mathrm{lip}}\right),
\end{aligned}
$$

and

$$
\frac{\partial q_{\mathrm{liq}}}{\partial t}=\underbrace{-u \frac{\partial q_{\mathrm{liq}}}{\partial r}-w \frac{\partial q_{\mathrm{liq}}}{\partial z}}_{\operatorname{ADV}\left(u, w, q_{\mathrm{liq}}\right)}+\operatorname{PHA}\left(\theta, q_{\mathrm{liq}}\right)+\text { rainfall. }
$$

The equations used in the adjoint model are as follows:

$$
\begin{aligned}
& \begin{array}{c}
-\frac{\partial \boldsymbol{\lambda}^{u}}{\partial t}=\underbrace{-\frac{\partial v}{\partial r} \boldsymbol{\lambda}^{v}-\frac{\partial u}{\partial r} \boldsymbol{\lambda}^{u}-u \frac{\partial \boldsymbol{\lambda}^{u}}{\partial r}-w \frac{\partial \lambda^{u}}{\partial z}-\frac{\partial w}{\partial r} \boldsymbol{\lambda}^{w}-\frac{\partial \theta}{\partial r}}_{\operatorname{ADV}^{*}\left(\boldsymbol{\lambda}^{u}, \boldsymbol{\lambda}^{v}, \boldsymbol{\lambda}^{w}, \boldsymbol{\lambda}^{\theta}, \boldsymbol{\lambda}^{q}, \boldsymbol{\lambda}^{q \mathrm{liq}}\right)} \\
-\frac{\partial \boldsymbol{\lambda}^{v}}{\partial t}=\underbrace{-u \frac{\partial \boldsymbol{\lambda}^{v}}{\partial r}-w \frac{\partial \boldsymbol{\lambda}^{v}}{\partial z}}_{\mathrm{ADV}^{*}\left(\boldsymbol{\lambda}^{v}\right)}-\underbrace{\frac{u}{r} \boldsymbol{\lambda}^{v}-\frac{2 v}{r} \boldsymbol{\lambda}^{u}}_{\operatorname{MET}^{*}\left(\boldsymbol{\lambda}^{u}, \boldsymbol{\lambda}^{v}\right)}+\underbrace{f \boldsymbol{\lambda}^{u}}_{\operatorname{COR}^{*}\left(\boldsymbol{\lambda}^{u}\right)},
\end{array} \\
& -\frac{\partial \boldsymbol{\lambda}^{w}}{\partial t}=\underbrace{-\frac{\partial v}{\partial r} \boldsymbol{\lambda}^{v}-\frac{\partial u}{\partial r} \boldsymbol{\lambda}^{u}-u \frac{\partial \boldsymbol{\lambda}^{w}}{\partial r}-w \frac{\partial \boldsymbol{\lambda}^{w}}{\partial z}-\frac{\partial w}{\partial z} \boldsymbol{\lambda}^{w}-\frac{\partial \theta}{\partial z}-\frac{\partial q}{\partial z} \boldsymbol{\lambda}^{q}-\frac{\partial q_{\mathrm{liq}}}{\partial z} \boldsymbol{\lambda}^{q_{\mathrm{liq}}}}_{\mathrm{ADV}^{*}\left(\boldsymbol{\lambda}^{u}, \boldsymbol{\lambda}^{v}, \boldsymbol{\lambda}^{w}, \boldsymbol{\lambda}^{\theta}, \boldsymbol{\lambda}^{q}, \boldsymbol{\lambda}^{q \text { liq }}\right)}+\operatorname{SMA}^{*}\left(\boldsymbol{\lambda}^{u}, \boldsymbol{\lambda}^{w}\right),
\end{aligned}
$$

$-\frac{\partial \boldsymbol{\lambda}^{\pi}}{\partial t}=\operatorname{PHA} *\left(\boldsymbol{\lambda}^{\theta}, \boldsymbol{\lambda}^{q}, \boldsymbol{\lambda}^{q_{\mathrm{liq}}}\right)+\mathrm{SMA}^{*}\left(\boldsymbol{\lambda}^{u}, \boldsymbol{\lambda}^{w}\right)$,

$$
\begin{aligned}
-\frac{\partial \boldsymbol{\lambda}^{\theta}}{\partial t}= & \underbrace{-u \frac{\partial \boldsymbol{\lambda}^{\theta}}{\partial r}-w \frac{\partial \boldsymbol{\lambda}^{\theta}}{\partial z}}_{\operatorname{ADV}^{*}\left(\boldsymbol{\lambda}^{\theta}\right)}+\underbrace{\frac{g}{\bar{\theta}} \boldsymbol{\lambda}^{w}}_{\operatorname{BUO}^{*}\left(\boldsymbol{\lambda}^{w}\right)} \\
& +\operatorname{PHA}^{*}\left(\boldsymbol{\lambda}^{q}, \boldsymbol{\lambda}^{q_{\text {liq }}}\right)+\operatorname{RAD}^{*}\left(\boldsymbol{\lambda}^{\theta}\right),
\end{aligned}
$$

$$
\begin{aligned}
-\frac{\partial \boldsymbol{\lambda}^{q}}{\partial t}= & \underbrace{-u \frac{\partial \boldsymbol{\lambda}^{q}}{\partial r}-w \frac{\partial \boldsymbol{\lambda}^{q}}{\partial z}}_{\mathrm{ADV}^{*}\left(\boldsymbol{\lambda}^{q}\right)} \underbrace{-0.61 g \boldsymbol{\lambda}^{w}}_{\mathrm{BUO}^{*}\left(\boldsymbol{\lambda}^{w}\right)} \\
& +\operatorname{PHA}^{*}\left(\boldsymbol{\lambda}^{\theta}, \boldsymbol{\lambda}^{q}, \boldsymbol{\lambda}^{\left.q_{\mathrm{liq}}\right)},\right.
\end{aligned}
$$

and

$$
\begin{aligned}
-\frac{\partial \boldsymbol{\lambda}^{q_{\text {liq }}}}{\partial t}= & \underbrace{-u \frac{\partial \boldsymbol{\lambda}^{q_{\text {liq }}}}{\partial r}-w \frac{\partial \boldsymbol{\lambda}^{q_{\text {liq }}}}{\partial z}}_{\operatorname{ADV}^{*}\left(\boldsymbol{\lambda}^{q_{\text {liq }}}\right)}+\underbrace{g \boldsymbol{\lambda}^{w}}_{\text {BUO }^{*}\left(\boldsymbol{\lambda}^{w}\right)} \\
& +\operatorname{PHA}^{*}\left(\boldsymbol{\lambda}^{\theta}, \boldsymbol{\lambda}^{q}\right)+\text { rainfall*. }
\end{aligned}
$$

Three-letter codes with asterisks represent the terms in the adjoint model corresponding to the terms in the forward model [Eqs. (A1)-(A7); Table 1]. For example, the term BUO* $\left(\lambda^{w}\right)$ in Eq. (A13) relates to the fact that the perturbation of $q$ at certain time affects later perturbation of $w$ through the buoyancy force term. We integrate these equations backward in time to obtain the sensitivity fields. The state variables $(u, v, w, \pi, \theta, q$, and $\left.q_{\text {liq }}\right)$ in the adjoint model are taken from the reference fields.

The sensitivity with respect to surface fluxes, SST, and air-sea exchange coefficients from a discretized individual time step $m$ to $m^{\prime}(>m)$ are given by

$$
\begin{aligned}
\boldsymbol{\lambda}_{r z}^{\tau_{r z}}(r)= & -\sum_{i=m}^{m^{\prime}} \frac{\Delta t}{\Delta z} \frac{\lambda_{l, i+1}^{u}(r)}{\rho}, \\
\boldsymbol{\lambda}^{\tau_{\phi z}}(r)= & -\sum_{i=m}^{m^{\prime}} \frac{\Delta t}{\Delta z} \frac{\lambda_{l, i+1}^{v}(r)}{\rho}, \\
\lambda^{\mathbf{F}_{H}(r)=}- & -\sum_{i=m}^{m^{\prime}} \frac{\Delta t}{\pi \rho C_{p} \Delta z} \lambda_{l, i+1}^{\theta}(r), \\
\lambda^{\mathbf{F}_{E}(r)=} & -\sum_{i=m}^{m^{\prime}} \frac{\Delta t}{\rho L \Delta z} \lambda_{l, i+1}^{q}(r), \\
\lambda^{\mathrm{SST}}(r)= & -\sum_{i=m}^{m^{\prime}} \frac{\Delta t}{\Delta z}\left[C_{\mathrm{H}}|\mathbf{V}(r)| \boldsymbol{\lambda}_{l, i+1}^{\theta}(r)\right. \\
& \left.+C_{\mathrm{E}}|\mathbf{V}(r)| \mathbf{Y} * \boldsymbol{\lambda}_{l, i+1}^{q}(r)\right],
\end{aligned}
$$




$$
\begin{gathered}
\boldsymbol{\lambda}^{C_{D}(r)=}=-\sum_{i=m}^{m^{\prime}} \frac{\Delta t|\mathbf{V}(r)|}{\Delta z}\left[u_{l, i+1}(r) \boldsymbol{\lambda}_{l, i+1}^{u}(r)\right. \\
\left.+v_{l, i+1}(r) \boldsymbol{\lambda}_{l, i+1}^{v}(r)\right], \\
\boldsymbol{\lambda}^{C_{H}(r)=-} \sum_{i=m}^{m^{\prime}} \frac{\Delta t|\mathbf{V}(r)|}{\Delta z}\left[\theta_{l, i+1}(r)-\theta_{s, i+1}\right] \boldsymbol{\lambda}_{l, i+1}^{\theta}(r),
\end{gathered}
$$

and

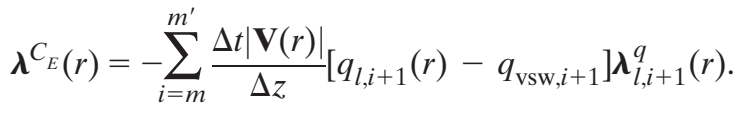

Here $i$ is the numbered time step, $\Delta t$ is the long time step, $\Delta z$ is the vertical grid spacing, and $q_{\mathrm{vsw}}$ is the saturated mixing ratio of water vapor at the sea surface. The operator $\mathbf{Y}$ represents the tangent linear operator for Tetens' equation when the temperature is equivalent to SST; that is, $\delta q=\mathbf{Y} \delta(\mathrm{SST})$ and $\mathbf{Y}^{*}$ is its adjoint. The subscripts $l$ and $s$ represent the corresponding values at the lowest atmospheric layer and at the sea surface, respectively.

\section{REFERENCES}

Bender, M., I. Ginis, R. Tuleya, B. Thomas, and T. Marchok, 2007: The operational GFDL coupled hurricane-ocean prediction system and a summary of its performance. Mon. Wea. Rev., 135, 3965-3989.

Bister, M., and K. Emanuel, 1998: Dissipative heating and hurricane intensity. Meteor. Atmos. Phys., 65, 233-240.

Black, P., and Coauthors, 2007: Air-sea exchange in hurricanes: Synthesis of observations from the Coupled Boundary Layer Air-Sea Transfer experiment. Bull. Amer. Meteor. Soc., 88, 357-374.

Bryan, G., and R. Rotunno, 2009a: The influence of near-surface, high-entropy air in hurricane eyes on maximum hurricane intensity. J. Atmos. Sci., 66, 148-158.

— and _ 2009b: The maximum intensity of tropical cyclones in axisymmetric numerical model simulations. Mon. Wea. Rev., 137, 1770-1789.

$\ldots$, and - 2009c: Evaluation of an analytical model for the maximum intensity of tropical cyclones. J. Atmos. Sci., 66, 3042-3060.

Donelan, M., W. Drennan, and K. Katsaros, 1997: The air-sea momentum flux in conditions of wind sea and swell. J. Phys. Oceanogr., 27, 2087-2099.

- B. Haus, N. Reul, W. Plant, M. Stiassnie, H. Graber, O. Brown, and E. Saltzman, 2004: On the limiting aerodynamic roughness of the ocean in very strong winds. Geophys. Res. Lett., 31, L18306, doi:10.1029/2004GL019460.

Doyle, J., C. Amerault, C. Reynolds, and J. Moskaitis, 2010: Initial condition sensitivity and predictability of tropical cyclogenesis. Extended Abstracts, 29th Conf. on Hurricanes and Tropical
Meteorology, Tucson, AZ, Amer. Meteor. Soc., P1.5. [Available online at http://ams.confex.com/ams/29Hurricanes/techprogram/ paper_168004.htm.]

Drennan, W., H. Graber, and M. Donelan, 1999: Evidence for the effects of swell and unsteady winds on marine wind stress. J. Phys. Oceanogr., 29, 1853-1864.

Emanuel, K., 1986: An air-sea interaction theory for tropical cyclones. Part I: Steady-state maintenance. J. Atmos. Sci., 43, 585-605.

_ 1995: Sensitivity of tropical cyclones to surface exchange coefficients and a revised steady-state model incorporating eye dynamics. J. Atmos. Sci., 52, 3969-3976.

- 2005: Increasing destructiveness of tropical cyclones over the past 30 years. Nature, 436, 686-688.

— C. DesAutels, C. Holloway, and R. Korty, 2004: Environmental control of tropical cyclone intensity. J. Atmos. Sci., 61, 843-858.

Errico, R., 1997: What is an adjoint model? Bull. Amer. Meteor. Soc., 78, 2577-2591.

Frank, W., and E. Ritchie, 2001: Effects of vertical wind shear on the intensity and structure of numerically simulated hurricanes. Mon. Wea. Rev., 129, 2249-2269.

Hausman, S., K. Ooyama, and W. Schubert, 2006: Potential vorticity structure of simulated hurricanes. J. Atmos. Sci., 63, 87-108.

Ito, K., Y. Ishikawa, and T. Awaji, 2010: Specifying air-sea exchange coefficients in the high-wind regime of a mature tropical cyclone by an adjoint data assimilation method. SOLA, 6, 13 16; Corrigendum, 6, E2.

Klemp, J., and R. Wilhelmson, 1978: The simulation of threedimensional convective storm dynamics. J. Atmos. Sci., 35, 1070-1096.

Knutson, T., C. Landsea, and K. Emanuel, 2010a: Tropical cyclones and climate change: A review. Global Perspectives on Tropical Cyclones: From Science to Mitigation, J. C. L Chan and J. D. Kepert, Eds., World Scientific, 243-286.

— Nat. Geosci., 3, 157-163.

Lewis, J., S. Lakshmivarahan, and S. Dhall, 2006: Dynamic Data Assimilation: A Least Squares Approach. Cambridge University Press, $654 \mathrm{pp}$.

Lin, I., C. Wu, K. Emanuel, I. Lee, C. Wu, and I. Pun, 2005: The interaction of Supertyphoon Maemi (2003) with a warm ocean eddy. Mon. Wea. Rev., 133, 2635-2649.

$\longrightarrow, \ldots$ I. Pun, and D. Ko, 2008: Upper-ocean thermal structure and the western North Pacific Category 5 typhoons. Part I: Ocean features and the Category 5 typhoons' intensification. Mon. Wea. Rev., 136, 3288-3306.

Lloyd, I., and G. Vecchi, 2011: Observational evidence for oceanic controls on hurricane intensity. J. Climate, 24, 1138-1153.

Miyamoto, Y., and T. Takemi, 2010: An effective radius of the sea surface enthalpy flux for the maintenance of a tropical cyclone. Atmos. Sci. Lett., 11, 278-282, doi:10.1002/asl.292.

Moon, I.-J., I. Ginis, T. Hara, and B. Thomas, 2007: A physicsbased parameterization of air-sea momentum flux at high wind speeds and its impact on hurricane intensity predictions. Mon. Wea. Rev., 135, 2869-2878.

Nordhaus, W., 2010: The economics of hurricanes and implications of global warming. Climate Change Econ., 1, 1-20.

Persing, J., and M. Montgomery, 2003: Hurricane superintensity. J. Atmos. Sci., 60, 2349-2371.

Powell, M., P. Vickery, and T. Reinhold, 2003: Reduced drag coefficient for high wind speeds in tropical cyclones. Nature, 422, 279-283. 
Price, J., 1981: Upper ocean response to a hurricane. J. Phys. Oceanogr., 11, 153-175.

$\longrightarrow$, T. Sanford, and G. Forristall, 1994: Forced stage response to a moving hurricane. J. Phys. Oceanogr., 24, 233-260.

Rotunno, R., and K. Emanuel, 1987: An air-sea interaction theory for tropical cyclones. Part II: Evolutionary study using a nonhydrostatic axisymmetric numerical model. J. Atmos. Sci., 44, 542-561.

Schade, L., and K. Emanuel, 1999: The ocean's effect on the intensity of tropical cyclones: Results from a simple coupled atmosphere-ocean model. J. Atmos. Sci., 56, 642-651.

Scharroo, R., W. Smith, and J. Lillibridge, 2005: Satellite altimetry and the intensification of Hurricane Katrina. Eos, Trans. Amer. Geohphys. Union, 86, 366, doi:10.1029/2005EO400004.

Smith, R., M. Montgomery, and S. Vogl, 2008: A critique of Emanuel's hurricane model and potential intensity theory. Quart. J. Roy. Meteor. Soc., 134, 551-561.

Southern, R., 1979: The global socio-economic impact of tropical cyclones. Aust. Meteor. Mag., 27, 175-195.

Suzuki, N., Y. Toba, and S. Komori, 2010: Examination of drag coefficient with special reference to the windsea Reynolds number: Conditions with counter and mixed swell. J. Oceanogr., 66, 731-739.

Torn, R., and G. Hakim, 2008: Ensemble-based sensitivity analysis. Mon. Wea. Rev., 136, 663-677.

Wang, Y., and C. Wu, 2004: Current understanding of tropical cyclone structure and intensity changes-A review. Meteor. Atmos. Phys., 87, 257-278.
Willoughby, H., 1988: The dynamics of the tropical cyclone core. Aust. Meteor. Mag., 36, 183-191.

Wu, C., K. Chou, Y. Wang, and Y. Kuo, 2006: Tropical cyclone initialization and prediction based on four-dimensional variational data assimilation. J. Atmos. Sci., 63, 2383-2395.

_ J. Chen, P. Lin, and K. Chou, 2007a: Targeted observations of tropical cyclone movement based on the adjoint-derived sensitivity steering vector. J. Atmos. Sci., 64, 2611-2626.

- C. Lee, and I. Lin, 2007b: The effect of the ocean eddy on tropical cyclone intensity. J. Atmos. Sci., 64, 3562-3578.

Xu, J., and Y. Wang, 2010: Sensitivity of tropical cyclone inner-core size and intensity to the radial distribution of surface entropy flux. J. Atmos. Sci., 67, 1831-1852.

Xu, Q., 1996: Generalized adjoint for physical processes with parameterized discontinuities. Part I: Basic issues and heuristic examples. J. Atmos. Sci., 53, 1123-1142.

_ 1997: Generalized adjoint for physical processes with parameterized discontinuities. Part IV: Problems in time discretization. J. Atmos. Sci., 54, 2722-2728.

Yamada, Y., K. Oouchi, M. Satoh, H. Tomita, and W. Yanase, 2010: Projection of changes in tropical cyclone activity and cloud height due to greenhouse warming: Global cloud-systemresolving approach. Geophys. Res. Lett., 37, L07709, doi:10.1029/ 2010 GL042518.

Yokoi, S., and Y. Takayabu, 2009: Multi-model projection of global warming impact on tropical cyclone genesis frequency over the western North Pacific. J. Meteor. Soc. Japan, 87, $525-538$. 